\title{
Effect of Tensor Representations for High-order Turbulence Correlations in Complex Axi-symmetric Flow Fields
}

\author{
M. Z. Afsar* \\ Department of Mechanical \& Aerospace Engineering, University of Strathclyde, Glasgow, G1 I XJ, UK. \\ V. Gryazev ${ }^{\dagger}$ \\ School of Engineering and Material Science, Queen Mary University of London, Mile End Road, London, E1 4NS, UK.
}

A. Markesteijn $\ddagger$

School of Engineering and Material Science, Queen Mary University of London, Mile End Road, London, E1 4NS, UK.

S. Karabasov $\S$

School of Engineering and Material Science, Queen Mary University of London, Mile End Road, London, E1 4NS, UK.

Dual-stream flows are common feature of turbofan engines in civil aviation. For jet flows with a significant (i.e. proportional of total mass flow) bypass stream, transport of momentum through mixing between streams may cause an apparent departure of axi-symmetric turbulence conditions. One of the pertinent features of such departure is the difference in amplitude of transverse diagonal components of velocity and Reynolds stress based correlation functionthat is correlation tensors of rank 2 and 4 respectively. Since these functions play a direct role in, among other things, the sound generation process, their accurate representation into an irreducible tensor form is vital in Engineering analysis. This paper builds on our previous work (AIAA 2020-2573, [1]) in which we assessed the kinematic structure of generalized auto-covariance tensor using turbulence data obtained by highly resolved Large-Eddy Simulations of complex dual stream jets. Now, however, we extend this work further by showing how a generalized form of the axisymmetric representation theory of the appropriate tensor correlation functions can be determined by exploiting the experimental observation that such correlation metrics are localized in small azimuthal separations for axisymmetric jets. The paper summarizes the new theory and shows initial comparisons of an appropriate tensor form using LES data reported in our previous work.

\section{Introduction}

$\mathrm{T}$ HE dual-stream jet flow represents the normal operating condition for most civil aircraft in which two co-axial streams emerge from the exit flow. The core flow is usually heated and of higher speed than the bypass flow, which is unheated and slower than the main stream. The breakdown of the shear layers emerging from the nozzle exit plane is complicated by large-scale mixing that takes places between both flows. The aim of this paper is to continue the work initiated in AIAA 2020-2573 [1] where we used a general set of theoretical criteria to assess the axi-symmetric structure of the exiting turbulent flow field. In the main, the auto-correlation of the high-order turbulence statistics possesses remains approximately axi-symmetric in the sense that the invariants formed by this tensor are, more-or-less, the same when they approximated by supposing the appropriate definition of axi-symmetric turbulence. In this paper, we extend this work to consider two pertinent effects. (1). We show that while the amplitudes of the said correlation functions (i.e. the auto-correlations) remain approximately axi-symmetric following the group-theoretic definition of this, the space-time structure of the tensor can show wide variation from this definition. In other words, at a fixed time-delay and/or fixed vector spatial separation (usually the streamwise is selected owing to the fact that the correlation lengths in this direction are larger than that in the transverse directions) the normalized correlation can depart from its value predicted when supposing that its scalar form remains invariant possesses an appropriate rotation group symmetry and

\footnotetext{
*Chancellor's Fellow. AIAA Member.

$\dagger$ Post-doctoral Research Associate. AIAA Member.

${ }^{\ddagger}$ Research Scientist / Director, GPU-prime Ltd. AIAA Member.

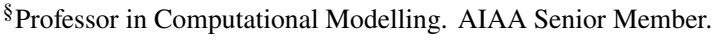




$\begin{array}{lllll}\text { Operating Point (OP) } & \text { Description } & M_{J} & T R & M a \\ \text { OP 1.3 } & \text { Dual stream heated ideally expanded } & 0.86 & 2.7 & 1.4 \\ \text { OP 1.7 } & \text { Single stream unheated subsonic } & 0.64 & 1.0 & 0.64\end{array}$

Table 1 Gryazev et al (2019) [2] test cases

therefore its components are related in some manner. (This could be in the form of the generalized Millionshchikov identity for example). (2). We show that effect of introducing a non-axi symmetric approximation to the Reynolds stress auto-covariance.

As in AIAA 2020-2573 [1], we use data obtained from a Large-Eddy Simulations of a high Reynolds number $\left(O\left(10^{6}\right)\right)$ heated and non-heated co-axial round jet flow that operates at conditions shown in table 1 . The large-eddy simulation data was reported in previously in Gryazev et al. (2019) and involves two static single-stream co-axial jets as depicted in Fig. 1. The LES calculation is based on the Monotonically Integrated LES (MILES) approach. The calculation domain includes the axi-symmetric nozzle geometry as well as the jet flow with grid volume of almost 20 million cells of the implicit-multi-block hexagonal type. For the calculations, the high-resolution CABARET method was used (see Semiletov et al. [3]).

\section{Definitions, symmetries and approximations}

Consider a region of non-homogeneous turbulence bounded within a high speed jet of order- 1 acoustic Mach number, $M a=U_{J} / c_{\infty}$ and order-1 temperature ratio, $T R$. Pressure fluctuations within the jet propagate to the far field where they are perceived as sound. We use Goldstein's generalized acoustic analogy [4] to represent this process in a manner whereby the wave propagation is calculated via a propagator tensor that depends on ALEE solution and the Reynolds stress auto-covariance tensor. The latter is modeled appropriately (see appendix). Let the pressure $p$, density $\rho$, enthalpy $h$, and speed of sound $c$ satisfy the ideal gas law equation of state $p=\rho c^{2} / \gamma$ and $h=c^{2} /(\gamma-1)$, where $\gamma$ denotes the ratio of specific heats.

The acoustic spectrum at the observation point, $\boldsymbol{x}=\left(x_{1}, \boldsymbol{x}_{T}\right)=\left(x_{1}, x_{2}, x_{3}\right)$, given by the Fourier transform

$$
I(\boldsymbol{x}, \omega) \equiv \frac{1}{2 \pi} \int_{-\infty}^{\infty} e^{i \omega \tau_{0}} \overline{p^{\prime}(\boldsymbol{x}, t) p^{\prime}\left(\boldsymbol{x}, t+\tau_{0}\right)} d \tau_{0},
$$

of the far-field pressure auto-covariance, $\overline{p^{\prime}(\boldsymbol{x}, t) p^{\prime}\left(\boldsymbol{x}, t+\tau_{0}\right)}$, can be expressed as a volume integral over a unit volume of turbulence at $\boldsymbol{y}=\left(y_{1}, \boldsymbol{y}_{T}\right)=\left(y_{1}, y_{2}, y_{3}\right)$ in the jet via

$$
I(\boldsymbol{x} ; \omega)=\int_{V_{\infty}(\boldsymbol{y})} I(\boldsymbol{x}, \boldsymbol{y} ; \omega) d \boldsymbol{y},
$$

where, $V_{\infty}(\boldsymbol{y})$ is the entire source region and the triple integral over the elemental volume $d \boldsymbol{y}$ is defined in Cartesian co-ordinates by $d \boldsymbol{y}=\prod_{n=1}^{3} d y_{n}$.

The pressure fluctuation in (1) is defined as $p^{\prime}(\boldsymbol{y}, \tau) \equiv p(\boldsymbol{y}, \tau)-\bar{p}(\boldsymbol{y})$ where over-bars are denote time average,

$$
\boldsymbol{\bullet}(\boldsymbol{x}) \equiv \lim _{T \rightarrow \infty} \frac{1}{2 T} \int_{-T}^{T} \bullet(\boldsymbol{x}, t) d t,
$$

such that $\bullet$ in $(3)$ is a place holder for any fluid mechanical variable, $T$ is the time period of averaging and, by definition, $\overline{\bullet^{\prime}}=0$.

Goldstein \& Leib (2008; hereafter referred to as G \& L) showed that $I(\boldsymbol{x}, \boldsymbol{y} ; \omega)$ on right side of 2 ) depends on the turbulence through the scripted tensor, $\mathcal{H}_{\lambda j \mu l}(\boldsymbol{y}, \boldsymbol{\eta} ; \omega)$, which is related to the Fourier transform

$$
H_{\lambda j \mu l}(\boldsymbol{y}, \boldsymbol{\eta} ; \omega)=\frac{1}{2 \pi} \int_{-\infty}^{\infty} e^{-i \omega \tau_{0}} R_{\lambda j \mu l}\left(\boldsymbol{y}, \boldsymbol{\eta} ; \tau_{0}\right) d \tau_{0}
$$


of the generalized auto-covariance tensor,

$$
R_{\lambda j \mu l}\left(\boldsymbol{y}, \boldsymbol{\eta} ; \tau_{0}\right) \equiv \lim _{T \rightarrow \infty} \frac{1}{2 T} \int_{-T}^{T} e_{\lambda j}(\boldsymbol{y}, \tau) e_{\mu l}\left(\boldsymbol{y}+\boldsymbol{\eta}, \tau+\tau_{0}\right) d \tau
$$

of the stationary random function, $e_{\lambda j}(\boldsymbol{y}, \tau)=-\left[\rho v_{\lambda}^{\prime} v_{j}^{\prime}-\overline{\rho v_{\lambda}^{\prime} v_{j}^{\prime}}\right](\boldsymbol{y}, \tau)$, by the linear transformation $\mathcal{H}_{\lambda j \mu l}(\boldsymbol{y}, \boldsymbol{\eta} ; \omega):=$ $\epsilon_{\lambda j \sigma m} H_{\sigma m \gamma n}(\boldsymbol{y}, \boldsymbol{\eta} ; \omega) \epsilon_{\mu l \gamma n}$. Comparing (5.12) to (5.13) in G \& L (2008) and using appropriate outer products of unit tensors in suffixes $(\lambda, j, \sigma, m)$ allows definition of the tensor as, $\epsilon_{\lambda j \sigma m} \equiv \delta_{\lambda \sigma} \delta_{j m}-\delta_{\lambda j} \delta_{\sigma m}(\gamma-1) / 2$ in the linear relation for $\mathcal{H}_{\lambda j \mu l}$ above. The four-dimensional perturbation velocity, $v_{\lambda}^{\prime}(\boldsymbol{y}, \tau) \equiv v_{\lambda}(\boldsymbol{y}, \tau)-\tilde{v}_{\lambda}(\boldsymbol{y})$ in which $v_{\lambda}^{\prime}=v_{i}^{\prime}$ is the ordinary fluid velocity perturbation when suffix, $\lambda=i=(1,2,3)$, otherwise $v_{\lambda}^{\prime}=v_{4}^{\prime}$ is proportional to enthalpy fluctuation. The latter denotes $v_{4}^{\prime}:=(\gamma-1)\left(h^{\prime}+v^{\prime 2} / 2\right) \equiv\left(c^{2}\right)^{\prime}+(\gamma-1) v^{\prime 2} / 2$ where $h^{\prime}$ is the fluctuating static enthalpy and $\left(c^{2}\right)^{\prime}$ is the fluctuations in the sound speed squared such that $v_{4}^{\prime} /(\gamma-1)$ denotes the moving frame stagnation enthalpy fluctuation (discussed further in [5]).

The tensor $R_{\lambda j \mu l}\left(\boldsymbol{y}, \eta_{1}, \eta_{\perp} ; \tau_{0}\right)$ possesses 144 components $(3 \times 4 \times 3 \times 4)$, however, owing to its two pair symmetry property - inasmuch as $R_{i j k l}=R_{j i k l}$ and $R_{i j k l}=R_{i j l k}$ when $(\lambda, \mu)=(1,2,3)-$ not all of these are independent. Afsar et al. (2011) (see table 1 on p.2525 of their paper) show that 144 reduces to 63 independent components when these symmetries are taken into account. Further reducing this number of components then involves achieving an "irreducible representation" of the tensor using its symmetry approximations, such as isotropy for example.

In this paper, we use an axisymmetric turbulence model that is a much more realistic kinematic representation for jets and which reduces the 63 components to a manageable number. The approximation assumes that the transverse correlation lengths are small compared to that in the streamwise flow direction. This is a well founded assertion in jets (see, for example, Pokora \& McGuirk's measurements[] in Figs. (19-21) and also Fig. (10) of their conference paper, AIAA 2008 - 3028). Afsar et al. [6] used Pokora \& McGuirk's data to propose that $R_{\lambda j \mu l}\left(\boldsymbol{y}, \eta_{1}, \eta_{\perp} ; \tau\right)$ is an axisymmetric tensor where $\eta_{\perp}=\left|\boldsymbol{\eta}_{\perp}\right|$ and $\boldsymbol{\eta}_{\perp}=\left(\eta_{2}, \eta_{3}\right)$. The spectral equivalent of this (Lemma's 3.1 and 3.2 in Afsar[7]) requires that $\Phi_{\lambda j \mu l}\left(\boldsymbol{y}, k_{1}, k_{\perp}^{2} ; \omega\right)$ is axisymmetric with the streamwise direction, $k_{1}$, being the principle direction of invariance. This tensor is defined below; see (19). The physical space approximation is consistent with experiments by Morris \& Zaman [? ] who show in their Fig. (15) that the transverse and azimuthal correlation lengths are virtually constant across range, $S t=(0.01-1.0)$ for an isothermal axisymmetric jet. The real space (i.e. non-spectral tensor) equivalent of Eq. (3.20) in reference [8] is:

$$
\begin{aligned}
R_{i j k l}\left(\boldsymbol{y}, \eta_{1}, \eta_{\perp} ; \tau_{0}\right) & =\left[\delta_{i j} \delta_{k l}-\delta_{i 1} \delta_{j 1} \delta_{k l}-\delta_{k 1} \delta_{l 1} \delta_{i j}+\delta_{i 1} \delta_{j 1} \delta_{k 1} \delta_{l 1}\right] R_{2222}\left(\boldsymbol{y}, \eta_{1}, \eta_{\perp} ; \tau_{0}\right) \\
& +\left[\delta_{i k} \delta_{j l}+\delta_{i l} \delta_{j k}-2 \delta_{i j} \delta_{k l}+2 \delta_{i 1} \delta_{j 1} \delta_{k l}+2 \delta_{k 1} \delta_{l 1} \delta_{i j}\right. \\
& \left.-\delta_{i 1} \delta_{l 1} \delta_{j k}-\delta_{j 1} \delta_{l 1} \delta_{i k}-\delta_{j 1} \delta_{k 1} \delta_{i l}-\delta_{i 1} \delta_{k 1} \delta_{j l}\right] R_{2323}\left(\boldsymbol{y}, \eta_{1}, \eta_{\perp} ; \tau_{0}\right) \\
& +\left[\delta_{i 1} \delta_{l 1} \delta_{j k}+\delta_{j 1} \delta_{l 1} \delta_{i k}+\delta_{j 1} \delta_{k 1} \delta_{i l}\right. \\
& \left.+\delta_{i 1} \delta_{k 1} \delta_{j l}-4 \delta_{i 1} \delta_{j 1} \delta_{k 1} \delta_{l 1}\right] R_{1212}\left(\boldsymbol{y}, \eta_{1}, \eta_{\perp} ; \tau_{0}\right) \\
& +\left[\delta_{i 1} \delta_{j 1} \delta_{k l}-\delta_{i 1} \delta_{j 1} \delta_{k 1} \delta_{l 1}\right] R_{1122}\left(\boldsymbol{y}, \eta_{1}, \eta_{\perp} ; \tau_{0}\right) \\
& +\left[\delta_{k 1} \delta_{l 1} \delta_{i j}-\delta_{i 1} \delta_{j 1} \delta_{k 1} \delta_{l 1}\right] R_{2211}\left(\boldsymbol{y}, \eta_{1}, \eta_{\perp} ; \tau_{0}\right) \\
& +\delta_{i 1} \delta_{j 1} \delta_{k 1} \delta_{l 1} R_{1111}\left(\boldsymbol{y}, \eta_{1}, \eta_{\perp} ; \tau_{0}\right)
\end{aligned}
$$

We can analyze the amplitude structure of $R_{i j k l}(y, 0 ; 0)$ in an obvious way that includes the complete set of 36 independent terms by computing the scalar (technically, quartilinear form), $R_{i j k l}(\boldsymbol{y}, 0 ; 0) R_{i j k l}(\boldsymbol{y}, 0 ; 0) / R_{1111}^{2}(\boldsymbol{y}, 0 ; 0)$. Comparison of the exact spatial distribution of this scalar to its reduction given by using (6) will then indicate, among other things, the relative importance of the 30 remaining independent components of $R_{i j k l}$ that are different from six primary (viz. axisymmetric) components: $\left(R_{1111}, R_{2222}, R_{1122}, R_{2211}, R_{1212}, R_{2323}\right)$, in the representation in Eq. 66.

Expanding the suffixes $(i, j, k, l)=(1,2,3)$ shows that the scalar form, $R_{i j k l}(\boldsymbol{y}, 0 ; 0) R_{i j k l}(\boldsymbol{y}, 0 ; 0)$, expands into 5 different groups involving square components inasmuch as

$$
R_{i j k l}(y, 0 ; 0) R_{i j k l}(y, 0 ; 0)=\sum_{m=1}^{7} P_{m} A_{m}\left(R_{1111}^{2}, R_{1111}^{2}, R_{1112}^{2}, R_{1113}^{2} \ldots, R_{i j k l}^{2}\right) .
$$


The numerical pre-factors in the set, $P_{m}=(1,1,1,2,4,4,8)$, can be expressed as $2^{r}$ where the base 2 is related to the fact there being an even multiplicity in the components of $R_{i j k l}$ owing to the pair symmetry at each point in the geometric configuration formed by the tensor in (5) at $\boldsymbol{y}$ and $\boldsymbol{y}+\boldsymbol{\eta}$ via $R_{i j k l}=R_{j i k l}$ and $R_{i j k l}=R_{i j l k}$. The two-pair symmetry property exists at non-zero space-time separation in (5) and reduces the number of independent components from $81(3 \times 3 \times 3 \times 3=81)$ to $36(6 \times 6=36)$. When $(\boldsymbol{\eta}, \tau)=0$ (and the auto-covariance tensor, $R_{i j k l}(\boldsymbol{y}, \boldsymbol{\eta}, \tau)$, reduces to its auto-correlation), 36 independent components reduces to 21 where $\sum_{m=1}^{7} P_{m}=21$. The latter reduction in number of components is because of the additional 'pair-interchange' symmetry where $R_{i j k l}=R_{k l i j}$ ensures all components above the leading diagonal of a $6 \times 6$ matrix of independent components is equal to the components below the diagonal (that runs from 1111 to 2323). The exponent, $r$, can then be formed by comparing the suffixes at $\boldsymbol{y}$ compared to $\boldsymbol{y}+\boldsymbol{\eta}$. That is, for the group of components for which $i \neq j$ or $k \neq l, r=n$ where $n$ is the minimum number of independent suffixes at $\boldsymbol{y}$ compared to $\boldsymbol{y}+\boldsymbol{\eta}$. E.g., for $R_{2212}, n=2 ; R_{2321}, n=3 ; R_{1212}, n=2$. The remaining components for which $i=j$ or $k=l, r=n-1$. E.g., for $R_{1111}, n=1$ and $r=0 ; R_{2233}, n=2$ and $r=1$. Expanding the tensor suffixes $(i, j, k, l)=(1,2,3)$ in (7) allows the coefficients $A_{m}\left(A_{1}, A_{2}, \ldots A_{5}\right)$ in (7) to be expressed by the the appropriate sum of squares of $R_{i j k l}$ inasmuch as:

$$
\begin{aligned}
R_{i j k l}(\boldsymbol{y}, 0 ; 0) R_{i j k l}(\boldsymbol{y}, 0 ; 0) & =\sum_{m=1}^{7} P_{m} A_{m}\left(R_{1111}^{2}, R_{1111}^{2}, R_{1112}^{2}, R_{1113}^{2} \ldots, R_{i j k l}^{2}\right) \\
& \rightarrow 1 \cdot\left[R_{1111}^{2}\right](\boldsymbol{y}, 0 ; 0) \\
& +1 \cdot\left[R_{2222}^{2}\right](\boldsymbol{y}, 0 ; 0) \\
& +1 \cdot\left[R_{3333}^{2}\right](\boldsymbol{y}, 0 ; 0) \\
& +2 \cdot\left[R_{1122}^{2}+R_{1133}^{2}+R_{2233}^{2}\right](\boldsymbol{y}, 0 ; 0) \\
& +4 \cdot\left[R_{1212}^{2}+R_{1313}^{2}+R_{2323}^{2}\right](\boldsymbol{y}, 0 ; 0) \\
& +4 \cdot\left[R_{1112}^{2}+R_{1113}^{2}+R_{1123}^{2}+R_{2212}^{2}\right. \\
& \left.+\quad R_{2213}^{2}+R_{2223}^{2}+R_{3312}^{2}+R_{3313}^{2}+R_{3323}^{2}\right](\boldsymbol{y}, 0 ; 0) \\
& +8 \cdot\left[R_{1213}^{2}+R_{2313}^{2}+R_{2321}^{2}\right](\boldsymbol{y}, 0 ; 0)
\end{aligned}
$$

Normalizing 8 by $R_{1111}^{2}$ gives a formula for scalar, $R_{i j k l}(y, 0 ; 0) R_{i j k l}(y, 0 ; 0)$, that we compare against its axi-symmetric reduction. That is, we compare:

$$
\begin{aligned}
{\left[\frac{R_{i j k l} R_{i j k l}}{R_{1111}^{2}}\right](y, 0 ; 0) } & =1+\left[\frac{R_{2222}^{2}+R_{3333}^{2}}{R_{1111}^{2}}\right](y, 0 ; 0) \\
& +2 \cdot\left[\frac{R_{1122}^{2}+R_{1133}^{2}+R_{2233}^{2}}{R_{1111}^{2}}\right](y, 0 ; 0) \\
& +4 \cdot\left[\frac{R_{1212}^{2}+R_{1313}^{2}+R_{2323}^{2}}{R_{1111}^{2}}\right](y, 0 ; 0) \\
& +4 \cdot\left[\frac{R_{1112}^{2}+R_{1113}^{2}+R_{1123}^{2}+R_{2212}^{2}+R_{2213}^{2}+R_{2223}^{2}+R_{3312}^{2}+R_{3313}^{2}+R_{3323}^{2}}{R_{1111}^{2}}\right](y, 0 ; 0) \\
& +8 \cdot\left[\frac{R_{1213}^{2}+R_{2313}^{2}+R_{2321}^{2}}{R_{1111}^{2}}\right](y, 0 ; 0)
\end{aligned}
$$

to its axi-symmetric reduction where $R_{i j k l}(\boldsymbol{y}, \boldsymbol{\eta}, \tau)$ is approximated by (6) and fourth and fifth square brackets in (9) are identically zero. In addition, $R_{2222}=R_{3333} \& R_{1212}=R_{1313}$ (axi-symmetry) and $R_{1122}=R_{2211}$ (pair interchange symmetry when $(\boldsymbol{\eta}, \tau)=0)$. Hence, $(9$ ) reduces to:

$$
\left[\frac{R_{i j k l} R_{i j k l}}{R_{1111}^{2}}\right](\boldsymbol{y}, 0 ; 0)=1+\left[\frac{2 \cdot R_{2222}^{2}+4 \cdot\left(R_{1122}^{2}+\frac{1}{2} R_{2233}^{2}\right)+8 \cdot\left(R_{1212}^{2}+\frac{1}{2} R_{2323}^{2}\right)}{R_{1111}^{2}}\right](\boldsymbol{y}, 0 ; 0)
$$



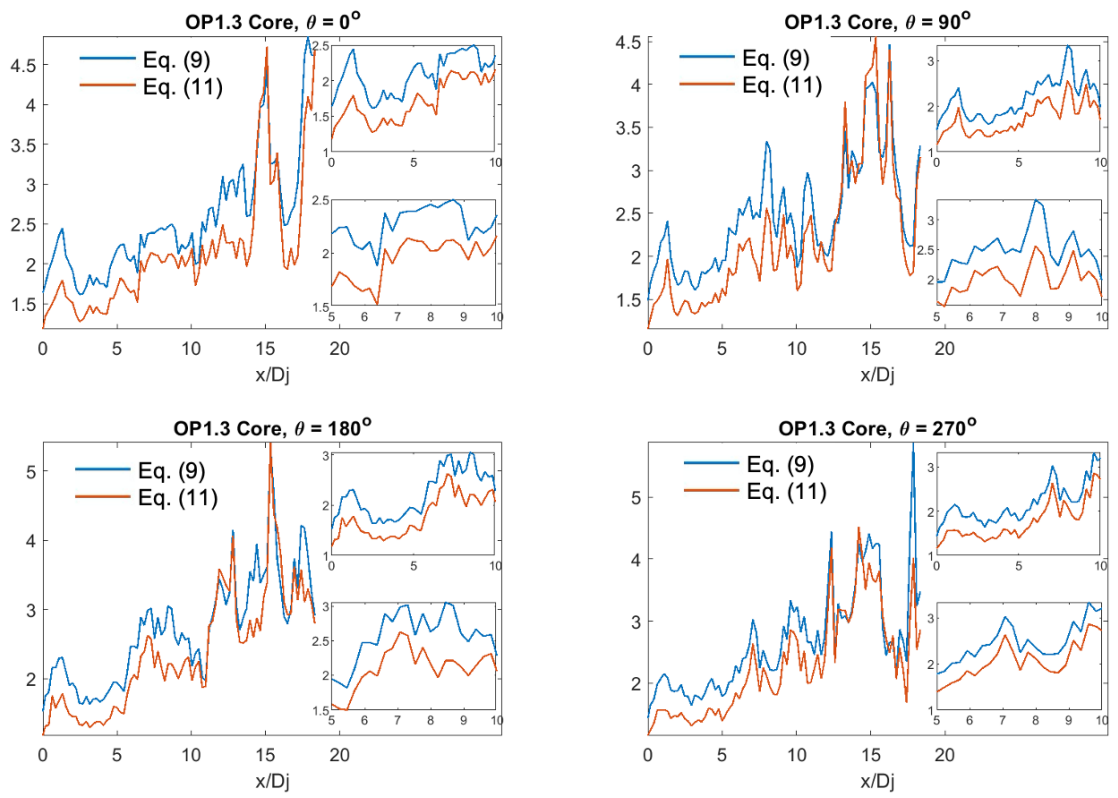

Fig. 1 Axial variation of square norm $R_{i j k l}^{2} / R_{1111}^{2}$ given by Eqs. (9) (LES data - no approximation) and (11) (LES data - axi-symmetry given by 6 ) for OP1.3 at the core shear layer radial location, $r / D_{J}=0.18$.

We can further simplify this expression by taking the square of the Millionshchikov identity, inasmuch as $R_{2222}^{2}=$ $\left(R_{2233}+2 R_{2323}\right)^{2}$, and inserting this latter result into 10, , to give

$$
\left[\frac{R_{i j k l} R_{i j k l}}{R_{1111}^{2}}\right](y, 0 ; 0)=1+\frac{R_{2323}^{2}}{\left(R_{1111} / 2\right)^{2}}\left[\left(1+\frac{R_{2233}}{R_{2323}}\right)^{2}+2 \cdot\left(1+\frac{R_{1212}^{2}+R_{1122}^{2}}{R_{2323}^{2}}\right)\right](y, 0 ; 0)
$$

Since Figs. (10a) \& (10b) in [9] show that amplitude ratio $R_{2233} / R_{2323} \approx 0$ along the shear layer at the streamwise location of the end of jet potential core (the acoustic Mach number in this case is 0.75 and the flow is isothermal). The amplitudes (of the auto-correlations) at $y_{1} / D_{J}=4 \& 6$ in Fig.10 of [9] appear as a ratio of $R_{1111}: R_{2233} / R_{1111}$ being almost zero at these locations and $R_{2323 / R_{1111}}$ between $(0.15-0.18)$. Hence, $[11)$ can be approximated by the deviatoric components $R_{1212}$ and $R_{2323}$ related to the in-plane and out-of-plane shear (i.e. in the $1-2$ co-ordinate plane and the perpendicular, $2-3$ plane). That is:

$$
\left[\frac{R_{i j k l} R_{i j k l}}{R_{1111}^{2}}\right](y, 0 ; 0)=1+\frac{R_{2323}^{2}}{\left(R_{1111} / 2\right)^{2}}\left[1+2 \cdot\left(1+\frac{R_{1212}^{2}}{R_{2323}^{2}}\right)\right](y, 0 ; 0),
$$

(where we have made use of the results in Fig. 10 in [9] and Fig. 6.6 in Afsar (2008) showing auto-correlation amplitude $R_{1122} / R_{1111}=R_{2211} / R_{1111}$ remains less than 0.1 at the end of the jet potential core). LES data of $R_{i j k l}$ components usually appears normalized with respect to largest component, $R_{1111}$. We can re-write (12) slightly in this as follows: $\tilde{R}_{i j k l}^{2} \rightarrow 1+4 \tilde{R}_{2323}^{2} \cdot\left(3+\tilde{R}_{1212}^{2} / \tilde{R}_{2323}^{2}\right)$ where $\tilde{R}_{i j k l}(y, 0 ; 0)=R_{i j k l}(\boldsymbol{y}, 0 ; 0) / R_{1111}(\boldsymbol{y}, 0 ; 0)$ is the normalized auto-correlation amplitude component. The auto-correlation amplitude data in Figs. (10) in [9] and (6.6) in Afsar (2008) indicate that ratio $\tilde{R}_{1212} / \tilde{R}_{2323} \approx 2$ along the shear layer at $y_{1} / D_{J}$ equals 4 and 6 (where $\tilde{R}_{1212} / \tilde{R}_{2323} \equiv R_{1212} / R_{2323}$ ).

Figures 1 - 3 show the axial variation of the square norm $R_{i j k l} / R_{111}^{2}$ comparing (9) (LES data without approximation) to (11) where the axisymmetric representation (6) is used. We show this at 4 azimuthal locations $\psi=\left(0^{\circ}, 90^{\circ}, 180^{\circ}, 270^{\circ}\right)$ for OP1.3 (core/bypass) and OP1.7 (bypass). In general there is about a 10\% or more difference between axi-symmetric theory Eq. (11) and LES data. Further approximation given by 12 performs similarly across azimuthal circumference and for both OPs; see Figs. 46 

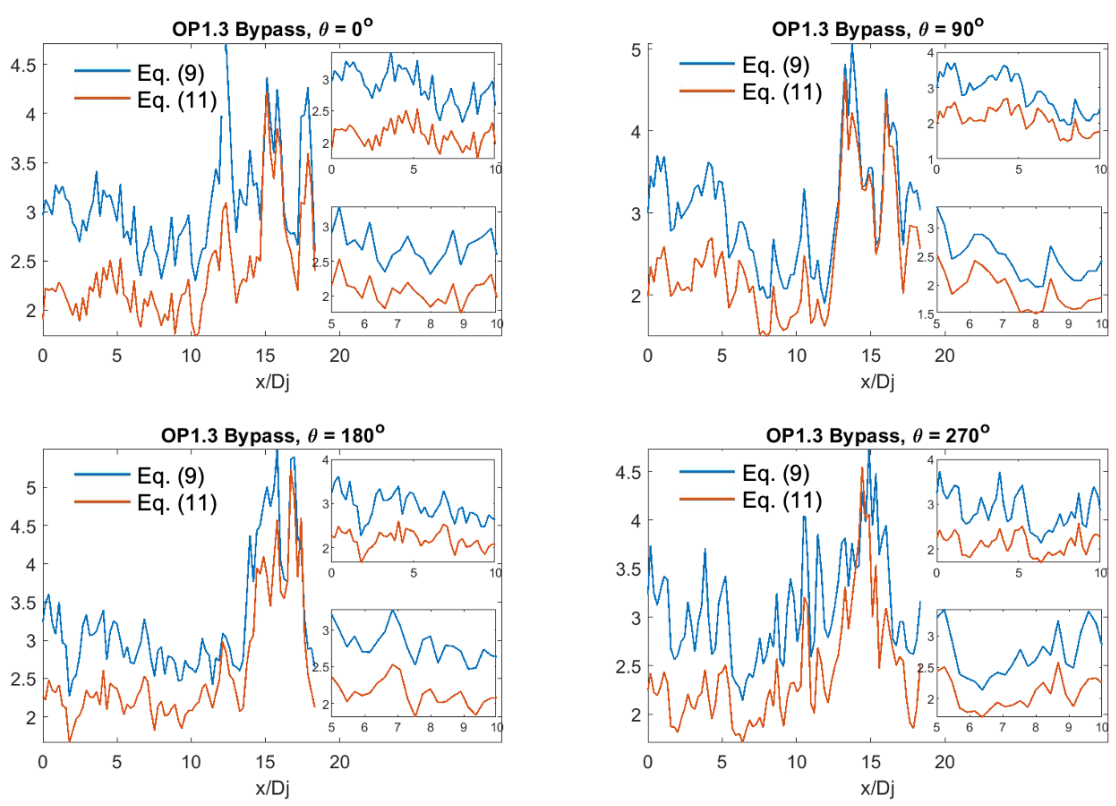

Fig. 2 Axial variation of square norm $R_{i j k l}^{2} / R_{1111}^{2}$ given by Eqs. [9] (LES data - no approximation) and (11) (LES data - axi-symmetry given by 6 ) for OP1.3 at the bypass shear layer radial location, $r / D_{J}=0.38$.

\section{Extended axisymmetry: reduction of Eq. (A.6) in [8]}

In reference [10] we show that the general expression for a rank-4 axi-symmetric tensor derived in [8] (Eq. A.6 in that paper) can be used to obtain an approximation for $R_{i j k l}\left(\boldsymbol{y}, \boldsymbol{\eta}, \tau_{0}\right)$ in which the number of independent components $\left(n_{c}\right)$ in the irreducible representation lies in the range $6 \leq n_{c} \leq 22$. In this way the new approximation includes further components beyond the 6 terms required by streamwise invariant representation in (A.9) of [8], given by (6) for the physical space auto-covariance tensor and, moreover, will allow examination of the importance of 'non-axisymmetric' components to the representation of (5).

Although the expansion below can be used on the full generalized auto-covariance tensor (i.e. on $R_{\lambda j \mu l}\left(\boldsymbol{y}, \boldsymbol{\eta} ; \tau_{0}\right)$ and its spectrum $\Phi_{\lambda j \mu l}(\boldsymbol{y}, \boldsymbol{k} ; \omega)$, we give the result only for the momentum flux or Reynolds stress auto-covariance associated manifold of the full tensor $R_{\lambda j \mu l}$ (or, $\Phi_{\lambda j \mu l}$ ) where the 4th component of the Greek suffixes, $(\lambda, \mu)$, is zero and $(\lambda, \mu)=(i, k)=(1,2,3)$. The momentum flux term enters term $I_{1}^{[1]}$ in the exact decomposition of the acoustic spectrum given by $(15)$ in [6]. The complete expansion of $\Phi_{\lambda j \mu l}(\boldsymbol{y}, \boldsymbol{k} ; \omega)$ involves determining the irreducible representation formed when the following quartilinear, trilinear and bilinear scalar forms $\left(\Phi_{i j k l} \hat{k}_{i} \hat{k}_{j} \hat{k}_{k} \hat{k}_{l}\right),\left(\Phi_{4 j k l} \hat{k}_{j} \hat{k}_{k} \hat{k}_{l}\right)$ and $\left(\Phi_{4 j 4 l} \hat{k}_{j} \hat{k}_{l}\right)$ remain invariant to the full rotation group at fixed $(\boldsymbol{y} ; \omega)=O(1)$ point/values. That is, determining the minimum number of permutations possible using the unit tensor (the alternating tensor is excluded for proper rotations of the vector configuration) and the independent vector arguments: $\boldsymbol{k}$ and the unit vector, $\lambda$. The latter vector represents a type of principal direction about which the vector configuration defined by the scalar forms is rotated and/or reflected for improper rotations. By the invariant theory, each permutation is multiplied by a scalar field that is an even function of vector arguments and can be related to components of the original spectral tensor. This appears in App. C of [6]. As explained in that paper, the invariant representation of the tensor $\Phi_{\lambda j \mu l}(\boldsymbol{y}, \boldsymbol{k} ; \omega)$ has to considered when the Greek suffixes take point values $(\lambda, \mu)=(4, i)$ and $(4,4)$ respectively because the pair symmetries property exists only when Greek suffixes are equal to Latin ones, $(\lambda, \mu)=(i, k)$ in $\Phi_{\lambda j \mu l}$ (i.e. the total number of objects in $\Phi_{\lambda j \mu l}$ is $3 \times 4 \times 3 \times 4=144$ reduces to $(6 \times 6+3 \times 6+3 \times 3)=63$; see Afsar et al. [6] for more details). While axi-symmetry considers the streamwise direction, $\lambda=\boldsymbol{e}_{1}$ to be the so-called principal direction about which the above scalar forms remain invariant (i.e. unaltered after rigid rotation), the authors of reference [6] showed that $\Phi_{\lambda j \mu l}$ depends on the transverse wavenumber vector, $\boldsymbol{k}_{\perp}$, through the square of its magnitude, $k_{\perp}^{2}=\left|\boldsymbol{k}_{\perp}\right|^{2}$ (Lemma 3.2 in [8]) when the physical space auto-covariance tensor, $R_{\lambda j \mu l}\left(\boldsymbol{y}, \boldsymbol{\eta} ; \tau_{0}\right)$ is approximated by its azimuthal average and, therefore, depends on the transverse correlation $\boldsymbol{\eta}_{\perp}$ only through the magnitude $\eta_{\perp}=\left|\boldsymbol{\eta}_{\perp}\right|$ (Eq. 16 in [6]). 

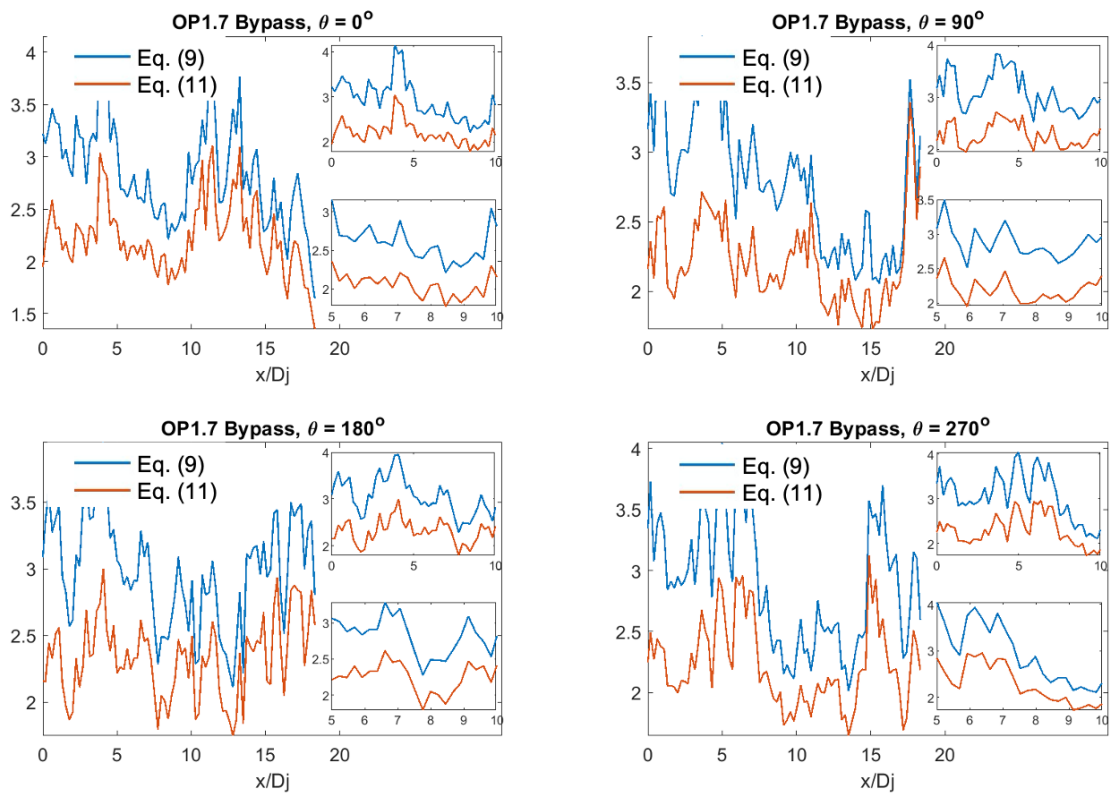

Fig. 3 Axial variation of square norm $R_{i j k l}^{2} / R_{1111}^{2}$ given by Eqs. (9) (LES data - no approximation) and (11) (LES data - axi-symmetry given by 6 ) for OP1.7 at the bypass shear layer radial location, $r / D_{J}=0.38$.

But the above approximation on $R_{\lambda j \mu l}\left(\boldsymbol{y}, \boldsymbol{\eta} ; \tau_{0}\right)$ follows automatically and self-consistently using simple asymptotic arguments. It has long been known that the transverse correlation length is an order of magnitude smaller than the streamwise for turbulence correlations in jet flows. This result was found by Pokora and McGuirk in water jets and subsequently confirmed by LES data in a high-speed isothermal air jet simulation; see Fig. 19b and cf. Fig. 20b in [11] for the $R_{1111}$ component at a streamwise location of $4 D_{J}$ downstream of the nozzle exit (this coincides with Harper-Bourne's measurements in [12]; p. 238 in [11]) along the shear layer at $r=0.5 D_{J}$.

The P\&M dataset shows that when $\eta_{1}$ is fixed, only very small increases of the $\eta_{2}$ result in a non-zero value for the $R_{1111}$ correlation component. P\&M indicate (p.241) that the streamwise separation, $\eta_{1}$ was non-zero in the initial auto-correlation measurement of $R_{1111}$ when $\eta_{2}=0$ (i.e. at the largest value of $R_{1111}$ which de-correlates in $\eta_{2}$ thereafter) to allow comparison with the hot-wire data in [12] and Davies et al. in [13] who took a similar initial $\eta_{1}$ value when $\eta_{2}$ was varied in $R_{1111}\left(\eta_{2}\right)$. As well as a reduction in magnitude, each correlation curve for $R_{1111}\left(\eta_{2}\right)$ in their Fig. 20 occurs at a much smaller value of the normalized separation $\left(\eta_{2}\right) / D_{J}$ compared to $R_{1111}\left(\eta_{1}\right)$ in P\&G's results. This extends to a maximum of $\left(\eta_{2}\right) / D_{J} \sim 0.078$ before $R_{1111}\left(\eta_{1}=\right.$ fixed, $\left.\eta_{2}\right) \rightarrow 0$ as $\tau_{0} \rightarrow \infty$ compared to $\left(\eta_{1}\right) / D_{J} \sim 0.6$ or so in Fig. 19b at which point $R_{1111}=R_{1111}\left(\eta_{1}, \eta_{2}=0\right) \rightarrow 0$ as $\tau_{0} \rightarrow \infty$. If $\eta_{1} / D_{J}=0.1$ in their measurements for the transverse auto-correlation, i.e. $R_{1111}\left(\eta_{1}, \eta_{2}, \tau_{0}\right)$ at $\eta_{2}=0$, then the intercept of normalized correlation, $R_{1111}$, on the ordinate axis is about 0.2 when $\left(\eta_{2}\right) / D_{J} \sim 0.039$. On the other hand, an intercept of the same magnitude (i.e. 0.2) in the streamwise correlation $R_{1111}\left(\eta_{1}, \eta_{2}=0\right)$ will occur at a streamwise separation $\eta_{1} / D_{J} \approx 0.3$. Hence the ratio of distances (streamwise to transverse) from where the correlation function reduces to the same normalized value is equal to $0.3 / 0.039 \sim 8$. In other words a reduction in correlation length from the transverse to the streamwise direction of order of 0.1. The second order correlation function shows similar trends in P\&M's data (Figs. 19a cf. 20a) but with a slight increase in the ratio above.

\section{A. Natural dependence of $R_{\lambda j \mu l}$ on a strained separation vector}

The above experimental findings can be naturally recovered when we suppose that $R_{\lambda j \mu l}\left(\boldsymbol{y}, \boldsymbol{\eta} ; \tau_{0}\right)$ depends on the separation $\boldsymbol{\eta}$ via the strained vector with components:

$$
\boldsymbol{\eta}:=\left\{\hat{\eta}_{1}, \epsilon \hat{\eta}_{2}, \epsilon^{2} \hat{\eta}_{3}\right\} l_{c} \equiv\left(\delta_{i 1} \hat{\eta}_{1}+\delta_{i 2} \epsilon \hat{\eta}_{2}+\delta_{i 3} \epsilon^{2} \hat{\eta}_{3}\right) l_{c}
$$



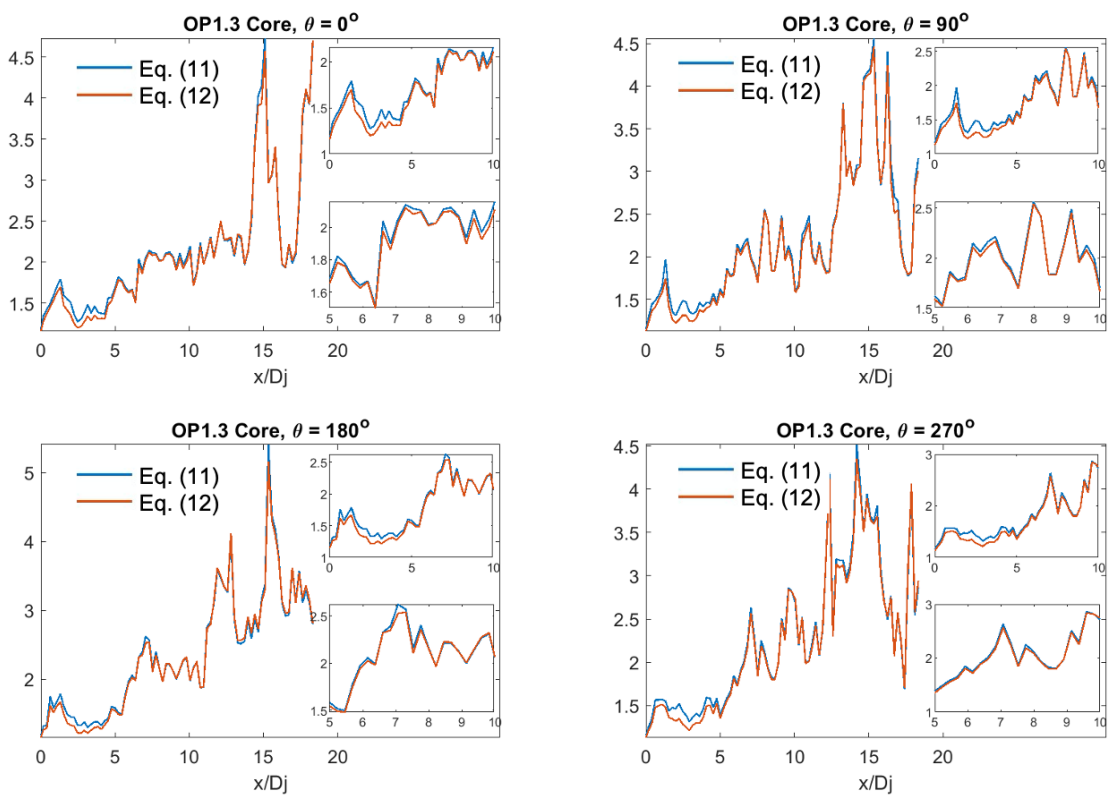

Fig. 4 Axial variation of square norm $R_{i j k l}^{2} / R_{1111}^{2}$ given by Eqs. 11 (LES data - axi-symmetry given by 6 ) and (12) (LES data - further approximation) for OP1.3 at the core shear layer radial location, $r / D_{J}=0.18$.

where $\delta_{i j}$ is the usual Kronecker delta function (unit tensor), $\left(\hat{\eta}_{1}, \hat{\eta}_{2}, \hat{\eta}_{3}\right)=O(1)$ and $\epsilon \ll O(1)$ (see reference [10]) and 13 possesses square-distance, $\hat{\eta}_{i}^{2}=\sum_{n=1}^{3} \epsilon^{2(n-1)} \hat{\eta}_{n}^{2}$. We suppose that the latter parameter (i.e. $\epsilon$ ) is asymptotically small in that it strains the separation vector in the arguments of the auto-covariance tensor, $R_{\lambda j \mu l}\left(\boldsymbol{y}, \boldsymbol{\eta} ; \tau_{0}\right)$ such that for an arbitrary increase in the normalized transverse separation $\hat{\eta}_{2}=O(1), \hat{R}_{\lambda j \mu l}\left(\boldsymbol{y}, \boldsymbol{\eta} ; \tau_{0}\right)$ is non-zero when $\eta_{2} / l_{c}=O(\epsilon)$ but is bounded at large time-delays inasmuch as $\hat{R}_{\lambda j \mu l} \rightarrow 0$ at any fixed $\boldsymbol{y}$ positioned in the turbulence energy-containing region of the jet when $\hat{\eta}_{(1,3)}=O(1)$. At values of $\eta_{2}$, (i.e., $\left.\eta_{2}>O(\epsilon)\right), \hat{R}_{\lambda j \mu l}\left(\boldsymbol{y}, \boldsymbol{\eta} ; \tau_{0}\right) \rightarrow 0$ for all $\hat{\tau}_{0}=O(1)$. P\&M's data shows that further increases in $\eta_{2}$ where $\eta_{2} \sim D_{J}$ (and of the same order as $\hat{\eta}_{1}=O(1)$ ) results in zero correlation; i.e., $\hat{R}_{\lambda j \mu l}\left(\boldsymbol{y}, \boldsymbol{\eta} ; \tau_{0}\right)=0$ in this region of the correlation volume. (This corresponds to the $\epsilon \rightarrow 0$ 'elongated eddy' limit discussed below Eq. 15. The normalized time delay here is defined as $\hat{\tau}_{0}=\tau_{0} U_{J} / D_{J}$ and $\hat{R}_{\lambda j \mu l}\left(\boldsymbol{y}, \boldsymbol{\eta} ; \tau_{0}\right)=R_{\lambda j \mu l}\left(\boldsymbol{y}, \boldsymbol{\eta} ; \tau_{0}\right) / R_{\lambda j \mu l}(\boldsymbol{y}, \mathbf{0} ; 0)$ (no sum across suffixes $(\lambda, j, \mu, l)$ in the latter ratio). Similar rapid de-correlation of $\hat{R}_{\lambda j \mu l}$ occurs with $\hat{\eta}_{3}=O(1)$ separations. That is, we assume via the scaling above in 13 that finite non-zero values of the normalized correlation function $\hat{R}_{\lambda j \mu l}$ is measured over short $\eta_{3} / l_{c}=O\left(\epsilon^{2}\right)$ separations. The data in Figs. (20b) \& (21b) in P\&M confirms this and we discuss its implication further below.

An alternative way to think about the dependence on the strained vector 13 in the rapid variation of $\hat{R}_{\lambda j \mu l}\left(\boldsymbol{y}, \boldsymbol{\eta} ; \tau_{0}\right)$ also implies that the normal/spanwise correlation lengths $\left(l_{c}^{(2)}, l_{c}^{(3)}\right)$ are much less than (i.e. asymptotically disparate with respect to) the streamwise correlation length; hence, $l_{c}^{(2)} \ll l_{c}^{(1)}$ and $l_{c}^{(3)} \ll l_{c}^{(1)}$. Using the definition of the radial length, $l_{c}^{(r)}=\sqrt{l_{c}^{(2) 2}+l_{c}^{(3) 2}}=\epsilon l_{c}+O\left(\epsilon^{2}\right)$, physically $\epsilon$ can now be defined more concretely as the ratio of the radial correlation length to streamwise correlation length; i.e. $\epsilon:=l_{c}^{(r)} / l_{c}=l_{c}^{(r)} / l_{c}^{(1)}$ since $\left\{l_{c}^{(1)}, l_{c}^{(2)}, l_{c}^{(3)}\right\}=l_{c}\left\{1, \epsilon, \epsilon^{2}\right\}$ by 13 and $l_{c}^{(i)}=\left\{\left\{l_{c}^{(1)}, l_{c}^{(2)}, l_{c}^{(3)}\right\}=l_{c}^{(i)}\left(\boldsymbol{y}, \tau_{0}\right)\right.$ is a function of $\boldsymbol{\eta}$ and therefore the rapid variation of $\hat{R}_{\lambda j \mu l}\left(\boldsymbol{y}, \boldsymbol{\eta} ; \tau_{0}\right)$ over the $(2,3) /(r, \phi)$-directions in the finite (bounded) correlation volume $V(\boldsymbol{\eta})$; Latin suffixes as before, $i=(1,2,3)$.

The length scale, $l_{c}$, has the upper bound: $\max \left\{l_{c}\right\}(\boldsymbol{y}, \boldsymbol{\eta}) \sim l_{\boldsymbol{\eta}}^{(1111}(\boldsymbol{y}, \boldsymbol{\eta})$ where $l_{\boldsymbol{\eta}}^{(1111}(\boldsymbol{y}, \boldsymbol{\eta})=\left|l_{\boldsymbol{\eta}}^{(1111)}\right|(\boldsymbol{y}, \boldsymbol{\eta})$ is the magnitude of the vector of correlation lengths, $\boldsymbol{l}_{\boldsymbol{\eta}}^{(1111)}(\boldsymbol{y}, \boldsymbol{\eta}):=\left(l_{\eta_{1}}^{(111)}, l_{\eta_{2}}^{(1111)}, l_{\eta_{3}}^{(1111)}\right)(\boldsymbol{y}, \boldsymbol{\eta})$, along the Cartesian directions $\boldsymbol{\eta}=\left(\eta_{1}, \eta_{2}, \eta_{3}\right)$ for the correlation function $R_{1111}\left(\boldsymbol{y}, \boldsymbol{\eta} ; \tau_{0}\right)$ (the largest component of $\hat{R}_{\lambda j \mu l}$, as mentioned above, defines the upper bound). The former (non-dimensional) length scale is defined by $(\lambda, j, \mu, l))=(1,1,1,1)$ 

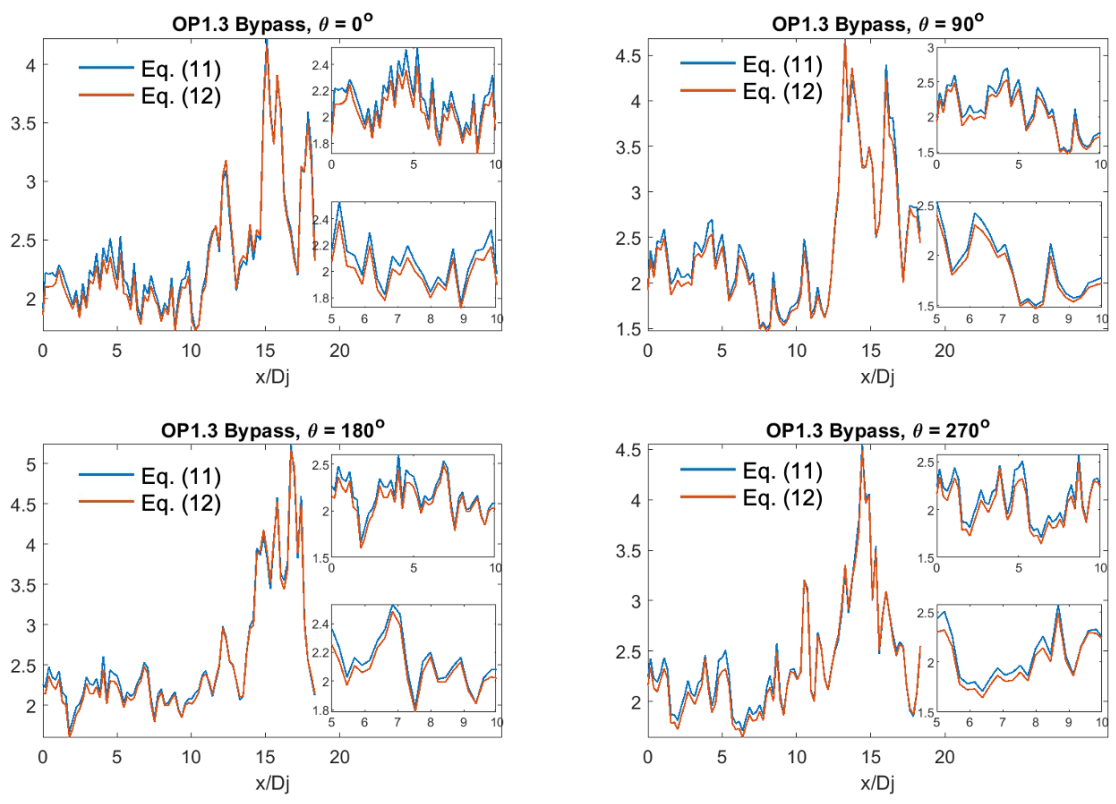

Fig. 5 Axial variation of square norm $R_{i j k l}^{2} / R_{1111}^{2}$ given by Eqs. 11 (LES data - axi-symmetry given by 6 ) and (12) (LES data - further approximation) for OP1.3 at the bypass shear layer radial location, $r / D_{J}=0.38$.

component of the integral over $\hat{\tau}_{0}$ :

$$
\boldsymbol{l}_{\boldsymbol{\eta}}^{(\lambda j \mu l)}(\boldsymbol{y}, \boldsymbol{\eta}):=\left\{l_{\eta_{1}}^{(\lambda j \mu l)}, l_{\eta_{2}}^{(\lambda j \mu l)}, l_{\eta_{3}}^{(\lambda j \mu l)}\right\}(\boldsymbol{y}, \boldsymbol{\eta})=\int_{0}^{\infty} \hat{R}_{\lambda j \mu l}\left(\boldsymbol{y}, \boldsymbol{\eta} ; \hat{\tau}_{0}\right) d \hat{\tau}_{0}
$$

The $(\lambda, j, \mu, l))=(1,1,1,1)$ component of the non-dimensional length scale, $l_{\eta}^{1111}=\left|l_{\eta}^{(1111)}\right|$, associated with the auto-covariance tensor $\hat{R}_{\lambda j \mu l}\left(\boldsymbol{y}, \boldsymbol{\eta} ; \hat{\tau}_{0}\right)$ lies between an upper bound defined by the jet diameter, $D_{J}$, and a lower bound (i.e., $l_{\eta}^{111}$ is much less than) the maximum acoustic wavelength, $\max \left(\lambda_{a c}\right) \sim O\left(k_{\infty}^{-1}\right)$ for the sound radiated at the lowest frequencies (where the non-dimensional frequency $\omega \ll 1$ ) representing the largest acoustic wavelengths. Hence, $l_{c} \sim l_{\eta}^{1111}$ is bounded by the inequality: $k_{\infty}^{-1} \ll l_{c} \leq D_{J}$ and can be taken as, $l_{c}=O(1)$, when the length scale defined by (14) is normalized by the fixed transverse spatial extent, $D_{J}$.

\section{B. Azimuthal dependence of $\hat{R}_{\lambda j \mu l}$ in the limit as $\epsilon \rightarrow 0$}

Three-component PIV measurements (3C-PIV)) of jet turbulence were conducted by P\&M (see [11], [14], [9], [3], [6] \& among others) to determine the appropriate correlation function components at various azimuthal separation angles at fixed streamwise/radial separations. In reference [8] the invariant representation of $\hat{R}_{\lambda j \mu l}\left(\boldsymbol{y}, \boldsymbol{\eta} ; \tau_{0}\right)$ was formulated for an arbitrary vector configuration of $(\boldsymbol{y}, \boldsymbol{\eta} ; \boldsymbol{\lambda})$ that reduced to Eq. (6) when $\boldsymbol{\lambda}=\boldsymbol{e}_{1}$ for turbulence evolving in a so-called 'cylindrical shell'. That is, at a fixed streamwise point, the azimuthal $(\Delta \psi)$ dependence of the auto-covariance tensor is neglected in a cylindrical interrogation volume that separates field points $\boldsymbol{y}$ and $z=(\boldsymbol{y}+\boldsymbol{\eta})$ (with $\boldsymbol{y}$ fixed in the definition given by Eq. 5] and the azimuthally averaged tensor assumed to be axi-symmetric; this is assumption 3.2 in [8].

In cylindrical co-ordinates, the field points are given by $\boldsymbol{y}=\left(y_{1}, r, \psi\right)$ and $z=\left(z_{1}, \tilde{r}, \tilde{\psi}\right)$ where radial locations are specified by $r^{2}:=|\boldsymbol{r}|^{2}=\left(y_{2}^{2}+y_{3}^{2}\right)$ and $\tilde{r}^{2}:=|\tilde{\boldsymbol{r}}|^{2}=\left(z_{2}^{2}+z_{3}^{2}\right)$ and azimuthal angles at these points are by $\tan \psi=y_{2} / y_{3}$ and $\tan \tilde{\psi}=z_{2} / z_{3}$. Moreover, at any streamwise location a vector in the $\boldsymbol{r}=\left(y_{2}, y_{3}\right)$ plane lies parallel to a vector in $\tilde{\boldsymbol{r}}=\left(z_{2}, z_{3}\right)$ plane in a manner such that the Jacobian for this isometry is unity: $\mathcal{J}(\boldsymbol{z}, \boldsymbol{\eta})=\prod_{i=1}^{3}\left|\partial z_{i} / \partial \eta_{i}\right|=1$ (i.e., a dilation). The separation vector that defines the cylindrical interrogation volume, $(\boldsymbol{z}-\boldsymbol{y})=\boldsymbol{\eta}$ is then specified uniquely by the triad, $\boldsymbol{\eta}=\left(\eta_{1}, \eta_{2}, \eta_{3}\right)$ in Cartesian co-ordinates and by $\boldsymbol{\eta}=\left(\eta_{1}, \eta_{\perp}, \Delta \psi\right)$ in this cylindrical co-ordinate system where $\eta_{1}=\left(z_{1}-y_{1}\right), \eta_{\perp}=(\tilde{r}-r)$ and $\Delta \psi=(\tilde{\psi}-\psi)$. But the latter assumption 3.2 introduced in [8] follows naturally 

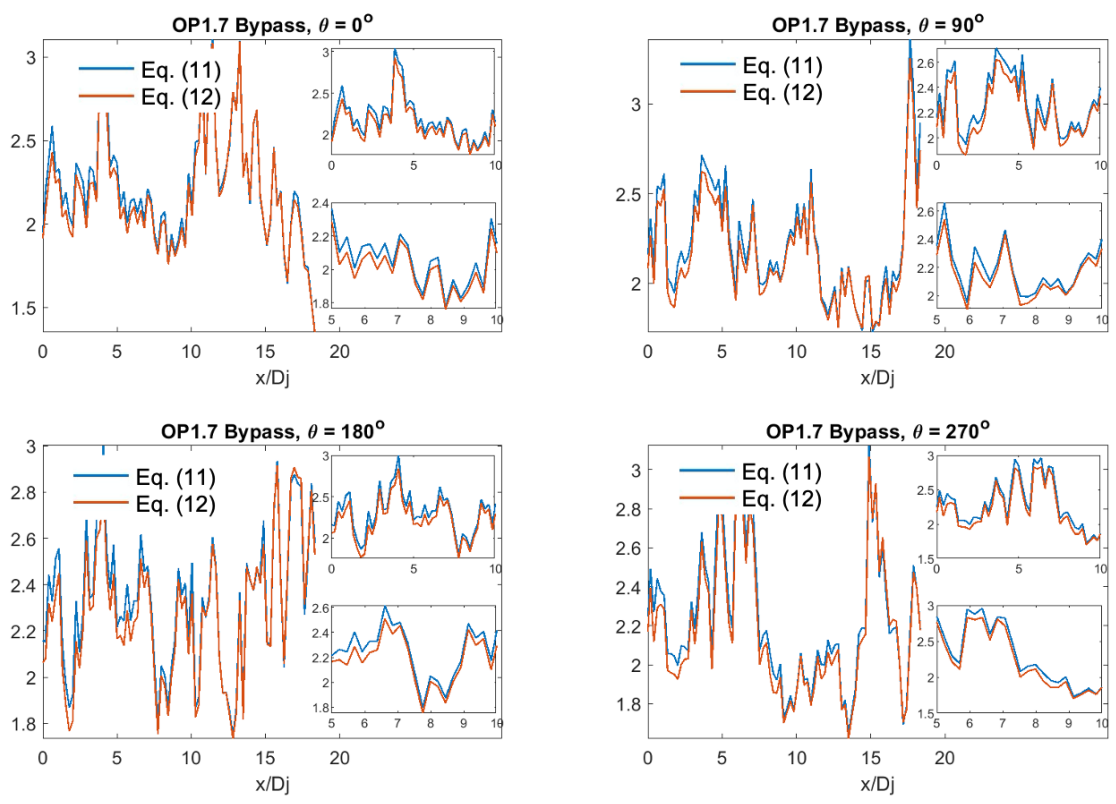

Fig. 6 Axial variation of square norm $R_{i j k l}^{2} / R_{1111}^{2}$ given Eqs. 111 (LES data - axi-symmetry given by 6 ) and (12) (LES data - further approximation) for OP1.7 at the bypass Shear layer radial location, $r / D_{J}=0.38$.

after inserting 13 into $\Delta \psi=\tan ^{-1}\left(\eta_{2} / \eta_{3}\right)$. and using the Taylor expansion formula (1.644 2) in Gradshetyn \& Ryzhik [15] gives the following expansion for the azimuthal angle, $\Delta \psi$ :

$$
\begin{aligned}
\Delta \psi\left(\eta_{2}, \eta_{3}\right) \equiv \Delta \psi\left(\hat{\eta}_{2}, \hat{\eta}_{3} ; \epsilon\right) & =\frac{\pi}{2}-\epsilon \cot \Delta \hat{\psi}+\frac{\epsilon^{3}}{3} \cot ^{3} \Delta \hat{\psi}+\ldots=\frac{\pi}{2}-\sum_{m=1}^{\infty}(-1)^{(m-1) / 2} \frac{\epsilon^{m}}{m} \cot ^{m} \Delta \hat{\psi} \\
& \rightarrow \frac{\pi}{2}-\epsilon \cot \Delta \hat{\psi}+O\left(\epsilon^{3}\right)
\end{aligned}
$$

where, after using 133 and the line below it, arbitrary variations in the re-scaled azimuthal angle $\Delta \hat{\psi}$ are defined via $\Delta \hat{\psi}=\tan ^{-1} \hat{\eta}_{2} / \hat{\eta}_{3}=O(1)$ and the integer $m$ in the summation formula in 15 takes on odd positive integral values: $m=2 n-1\left(n \in \mathcal{Z}^{+}\right)$. In this sense, $\Delta \psi\left(\hat{\eta}_{2}, \hat{\eta}_{3} ; \epsilon\right)$ expands as a Poincaré series with algebraic gauge functions in odd powers of the small parameter, $\epsilon$, multiplied by a pre-factors of the form: $\cot ^{m} \Delta \hat{\psi} / m$ for the $m$ 'th odd term in the series. That is, $\Delta \psi\left(\hat{\eta}_{2}, \hat{\eta}_{3} ; \epsilon\right)=O(1)-\epsilon \cot \Delta \hat{\psi}+\epsilon^{3}\left(\cot ^{3} \Delta \hat{\psi} / 3\right)+\ldots$ For angular separations where $\Delta \hat{\psi} \ll O(1)$, the remainder term in 15 expands like $\sim \epsilon / \Delta \hat{\psi}$ : the latter of which makes an asymptotically small $o(\epsilon / \Delta \hat{\psi})$ contribution to 15 in the limit as $\epsilon \rightarrow 0$.

More generally, the remainder term in (15) is easily bounded for variations in $\left(\hat{\eta}_{2} / \hat{\eta}_{3}\right)$ using formulas $(1.6442)$ and the Taylor expansion for $\cot \hat{\psi}$ in (1.411 7) in G\&R [15]. The $\epsilon$-prefactor is then bounded by the magnitude, $\left|\cot \tan ^{-1}\left(\hat{\eta}_{2} / \hat{\eta}_{3}\right)\right|$. But since, $\cot z=1 / z-z / 3+O\left(z^{3}\right)$, the next order term in 15 will remain $O(\epsilon)$ when $\tan ^{-1} z$ (that enters above) is expanded for $z=\left(\hat{\eta}_{2} / \hat{\eta}_{3}\right)=O(1)$ transverse separations. But still, in the limit as $\epsilon \rightarrow 0$, this contribution goes to zero like $o(\epsilon)$ in 15 when $\epsilon \rightarrow 0$ faster than the increase in $\cot \Delta \hat{\psi}$ for $O(1)$ changes in $\Delta \hat{\psi}$. Physically, this limit corresponds to turbulent structures (measured by the auto-covariance tensor in Eq. 5) that remain elongated (i.e. infinitely long) in the streamwise correlation length at lowest order in $\epsilon$ when $\Delta \psi \geq 0$ inasmuch as $l_{c} \gg O(1) \rightarrow \infty$ with $O(\epsilon)$ depth in the radial direction in which $\hat{\eta}_{\perp}$ expands as $\hat{\eta}_{\perp}=\sqrt{\eta_{\perp}^{2} / l_{c}^{2}}=\epsilon \hat{\eta}_{2}+O\left(\epsilon^{3}\right)$.

For long elongated streamwise turbulence structures that we are considering here, and for which $\epsilon \ll O(1)$ (or $\left.l_{c} \gg O(1) \rightarrow \infty\right)$, these simple asymptotic considerations can be interpreted by saying $\hat{R}_{\lambda j \mu l}\left(\boldsymbol{y}, \boldsymbol{\eta} ; \tau_{0}\right)$ remains azimuthally correlated over a relatively short range of angular separation, $\Delta \psi$; i.e., the value of the normalized correlation function will remain non-zero for only relatively small azimuthal angles (compared to $2 \pi$ - the latter is

\footnotetext{
*Simple co-ordinate geometry shows that $\Delta \psi=\tan ^{-1}\left(\eta_{2} / \eta_{3}\right)=\tan ^{-1}\left(z_{2}-y_{2}\right) /\left(z_{3}-y_{3}\right)$ is equivalent to $\Delta \psi=\tan ^{-1}\left(z_{2} / z_{3}\right)-\tan ^{-1}\left(y_{2} / y_{3}\right)$.
} 
the upper limit of the azimuthal average introduced in [8]). This is clear from Fig. 21b in P\&M's paper for the largest component $R_{1111}\left(y, \eta_{1}, \eta_{\perp}, \Delta \psi ; \tau_{0}\right)$. That is, they find that at an azimuthal angle of $\Delta \psi=10^{\circ}$, the normalized $R_{1111}\left(\boldsymbol{y}, \eta_{1}, \eta_{\perp}, \Delta \psi ; \tau_{0}\right)$ reduces by almost $\sim 80 \%$ (from 1.0 for the auto-correlation of $R_{1111}\left(\boldsymbol{y}, \boldsymbol{\eta} ; \tau_{0}\right)$ to $\sim 0.2$ at $\Delta \psi=10^{\circ}$ ); the amplitude at this $\Delta \psi$ is also very small, running from 0.2 to 0.1 across the range of normalized time delay (i.e., from $\tau_{0} U_{c} / D_{j}=0$ to approximately 5 respectively). At $\Delta \psi=20^{\circ}$, there is negligible variation of $R_{1111}\left(\boldsymbol{y}, \boldsymbol{\eta} ; \tau_{0}\right)$ across the range of time delay and the amplitude is basically at zero.

This data is consistent with Harper-Bourne's [12] turbulence measurements (the red dots in Fig. 21 in P\&M). Indeed, on p. 241, P\&M write that: "These azimuthal correlations (i.e., $R_{11}$ and $R_{1111}$ ) are significantly flatter than the axial or radial correlations for quite small increases in the separation vector". Note that, Fig. 15 in M\&Z's data shows the Fourier transform of the $R_{1111}\left(\boldsymbol{y}, \boldsymbol{\eta} ; \tau_{0}\right)$ possess an integral length scale that remains basically constant over a frequency range of covering the peak jet noise (that is $S t \sim(0.02-0.7)$ ) must be for values of $\Delta \psi<\Delta \psi_{0}$ where $\Delta \psi_{0} \neq 0^{\circ}$ is the minimum (non-zero) azimuthal angle above which $\hat{R}_{\lambda j \mu l}\left(\boldsymbol{y}, \eta_{1}, \eta_{\perp}, \Delta \psi ; \tau_{0}\right)$ de-correlates to zero for all time delays, $\tau_{0}$; i.e., $\hat{R}_{\lambda j \mu l}\left(\boldsymbol{y}, \eta_{1}, \eta_{\perp}, \Delta \psi>\Delta \psi_{0} ; \tau_{0}\right) \rightarrow 0 \forall \tau_{0} \in[0, \infty)$. (The " 0 " subscript indicates that the magnitude of the auto-covariance is finite at this maximum azimuthal separation which obviously lies between $\Delta \psi=0^{\circ}$ to $\Delta \psi \approx 20^{\circ}$; at the former (autocorrelation location) $\hat{R}_{\lambda j \mu l}=1$ and at the latter azimuthal angle $\hat{R}_{\lambda j \mu l}$ has de-correlated to 0 ). P\&M's data indicates that $\Delta \psi_{0} \approx 10^{\circ}$ or so.

Fig 7 indicates that for OP1.3 at the core shear layer location, the amplitude of $R_{1111}$ does not vary that significantly over the 4 azimuthal planes: $\psi=\left(0^{\circ}, 90^{\circ}, 1800^{\circ}, 2700^{\circ}\right)$. See Fig 8 also which shows the point values of the amplitude at the start and end of the potential core region for OP1.3. We might expect, therefore, that $R_{1111}$ will have a similar small variation in azimuthal separation $\Delta \psi$ in the core region. It is interesting that at the bypass radius for OP 1.3 and 1.7 in Fig. 7. the variation in amplitude for $R_{1111}$ is about $10 \%$ at the axial location, $x / D_{j}=6$; this is shown more clearly in the barcharts in Figs 8 \& 9 where the azimuthal variation appears to increase for OP1.3 at the bypass radius location further along the streamwise axis at the location $12 D_{j}$ (note that " $x / D_{j}$ " in the figures and captions corresponds to $y_{1} / D_{j}$ in the analysis).
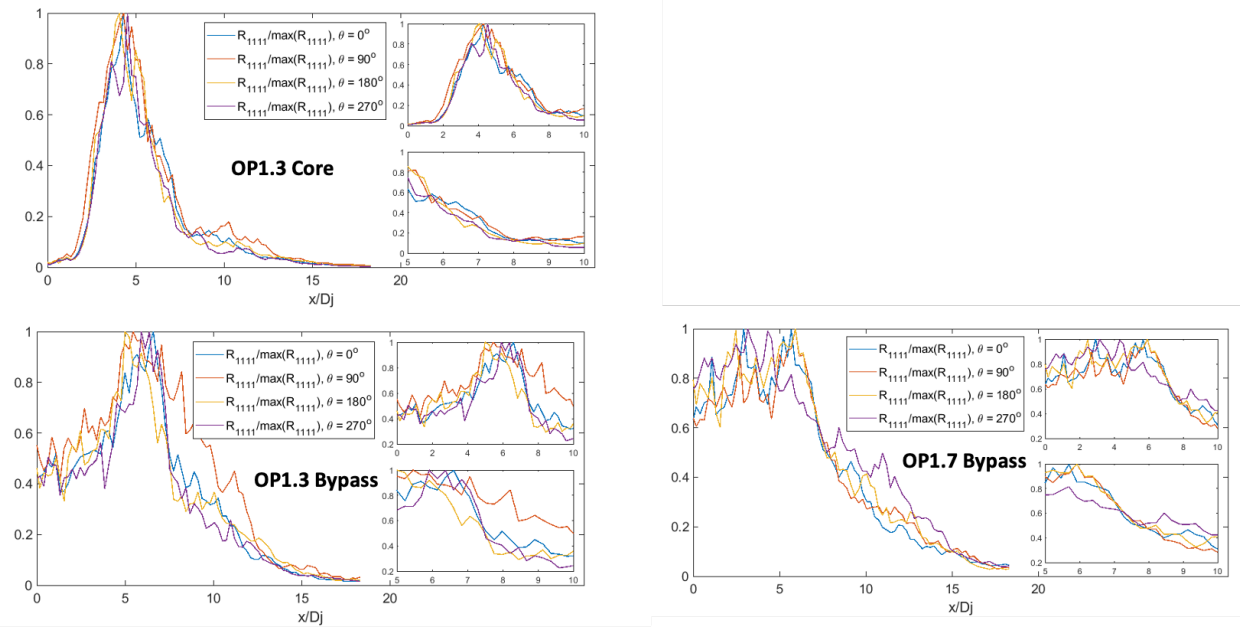

Fig. 7 Axial variation of $R_{1111}$ at 4 azimuthal planes: $\psi=\left(0^{\circ}, 90^{\circ}, 180^{\circ}, 270^{\circ}\right)$ for $\mathrm{OP} 1.3$ at the core/bypass shear layer radial locations $r / D_{j}=(0.18,0.38)$ respectively. OP1.7 is at the bypass radial location, $r / D_{J}=0.38$.

\section{An asymptotically exact 'cylindrical shell'}

The azimuthal average of $\hat{R}_{\lambda j \mu l}\left(\boldsymbol{y}, \eta_{1}, \eta_{\perp}, \Delta \psi ; \tau_{0}\right)$ over $\Delta \psi \in[0,2 \pi]$ reduces to the much shorter interval $\left[0, \Delta \psi_{0}\right]$ where $\Delta \psi_{0} \neq 0$ is a small non-zero wedge as P\&M and HB's data in [11, 12] both indicate. In ref. [10] we show that the azimuthal average:

$$
\bar{R}_{\lambda j \mu l}\left(\boldsymbol{y}, \eta_{1}, \eta_{\perp} ; \tau_{0}\right)=\frac{1}{2 \pi} \int_{0}^{2 \pi} \hat{R}_{\lambda j \mu l}\left(\boldsymbol{y}, \eta_{1}, \eta_{\perp}, \Delta \psi ; \tau_{0}\right) d \Delta \psi \rightarrow \frac{1}{\Delta \psi_{0}} \int_{0}^{\Delta \psi_{0}} \hat{R}_{\lambda j \mu l}\left(\boldsymbol{y}, \eta_{1}, \eta_{\perp}, \Delta \psi ; \tau_{0}\right) d \Delta \psi .
$$



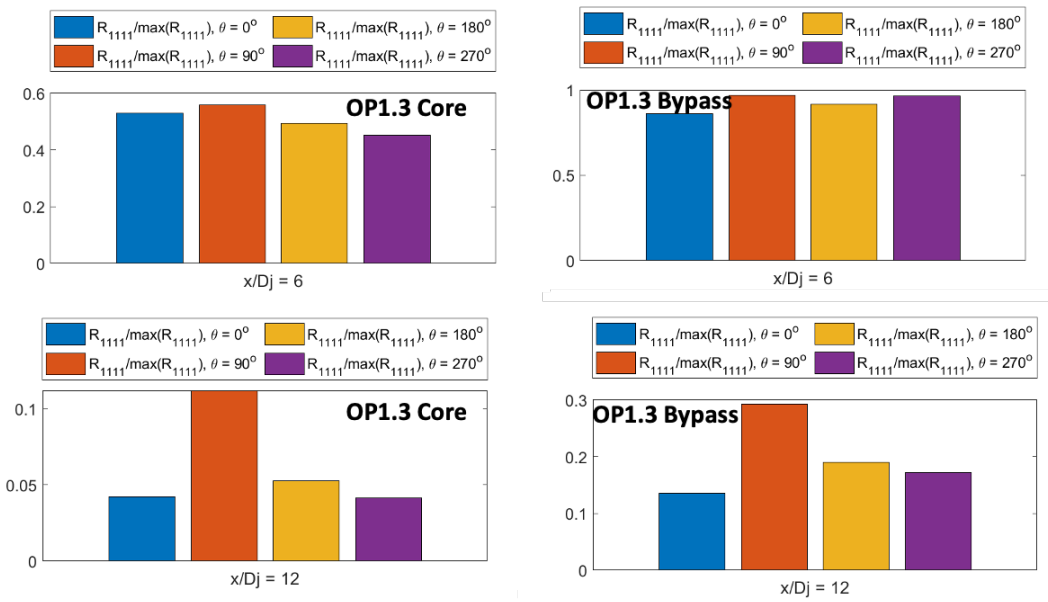

Fig. 8 Variation of $R_{1111}$ at 4 azimuthal planes: $\psi=\left(0^{\circ}, 90^{\circ}, 180^{\circ}, 270^{\circ}\right)$ for $\mathbf{O P} 1.3$ at the core/bypass shear layer radial locations $r / D_{j}=(0.18,0.38)$ respectively.
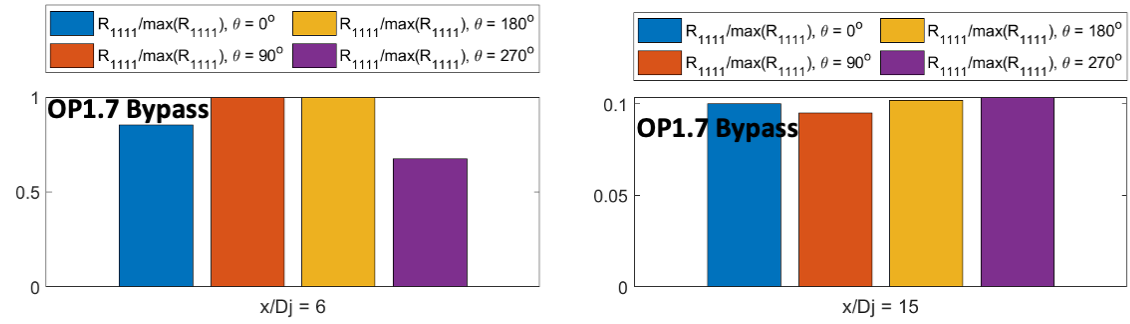

Fig. 9 Variation of $R_{1111}$ at 4 azimuthal planes: $\psi=\left(0^{\circ}, 90^{\circ}, 180^{\circ}, 270^{\circ}\right)$ for $\mathrm{OP} 1.7$ at the bypass shear layer radial locations $r / D_{j}=0.38$.

In refs. [8] and [6], $\hat{R}_{\lambda j \mu l}\left(\boldsymbol{y}, \eta_{1}, \eta_{\perp}, \Delta \psi ; \tau_{0}\right)$ was approximated to be independent of $\Delta \psi$ without rational justification. However, assumption (3.2) in ref.[8] now follows automatically when the streamwise and transverse turbulence length scales are asymptotically disparate and $\epsilon \rightarrow 0$. We show in ref.[10] that the lowest term in the Taylor expansion of $\hat{R}_{\lambda j \mu l}\left(\boldsymbol{y}, \eta_{1}, \eta_{\perp}, \Delta \psi ; \tau_{0}\right)$ in 17 is then formally azimuthally independent inasmuch as,

$$
\hat{R}_{\lambda j \mu l}\left(\boldsymbol{y}, \eta_{1}, \eta_{\perp}, \Delta \psi ; \tau_{0}\right)=\hat{R}_{\lambda j \mu l}\left(\boldsymbol{y}, \eta_{1}, \eta_{\perp}, 0 ; \tau_{0}\right)+o(1),
$$

to within an algebraically small remainder term in the limit as $\epsilon \rightarrow 0$. The azimuthal average of $\hat{R}_{\lambda j \mu l}\left(y, \eta_{1}, \eta_{\perp}, \Delta \psi ; \tau_{0}\right)$ in $(16)$ is therefore given by

$$
\bar{R}_{\lambda j \mu l}\left(\boldsymbol{y}, \eta_{1}, \eta_{\perp} ; \tau_{0}\right) \rightarrow \frac{1}{\Delta \psi_{0}} \int_{0}^{\Delta \psi_{0}} \hat{R}_{\lambda j \mu l}\left(\boldsymbol{y}, \eta_{1}, \eta_{\perp}, \Delta \psi ; \tau_{0}\right) d \Delta \psi=\hat{R}_{\lambda j \mu l}\left(\boldsymbol{y}, \eta_{1}, \eta_{\perp}, 0 ; \tau_{0}\right),
$$

where $\bar{R}_{\lambda j \mu l}\left(\boldsymbol{y}, \eta_{1}, \eta_{\perp} ; \tau_{0}\right) \equiv \bar{R}_{\lambda j \mu l}\left(\boldsymbol{y}, \hat{\eta}_{1}, \hat{\eta}_{\perp} ; \tau_{0}, \epsilon\right)$.

We validate the issue of azimuthal variation of $\hat{R}_{\lambda j \mu l}\left(\boldsymbol{y}, \eta_{1}, \eta_{\perp}, \Delta \psi ; \tau_{0}\right)$ in ref. [10] using LES data, for now we consider the effect of including the $O(\epsilon)$ correction to the representation in $(6)$ where $\bar{R}_{\lambda j \mu l}\left(y, \eta_{1}, \eta_{\perp} ; \tau_{0}\right)$ depends on $\hat{\eta}_{\perp}$ via $\hat{\eta}_{\perp}=\sqrt{\eta_{\perp}^{2} / l_{c}^{2}}=\epsilon \hat{\eta}_{2}+O\left(\epsilon^{3}\right)$. As shown in ref. [8], this is done more transparently using the spectral tensor because the shell theory models of turbulence (ch.3 in [16]) are derived from this starting point.

\section{Homogenization of the averaged spectral tensor in transverse wavenumber co-ordinates}

Following (3.1) in ref. [8], we assume that $\hat{R}_{\lambda j \mu l}\left(\boldsymbol{y}, \eta_{1}, \eta_{\perp}, \Delta \psi ; \tau_{0}\right)$ is an axi-symmetric tensor. This means that the scalar form defined by $\hat{R}_{\lambda j \mu l}$ is invariant to the full rotation group with respect to the streamwise unit vector, 
$\lambda=\boldsymbol{e}_{1}$. That is a 3D rotation/reflection of the vector configuration (connecting $\boldsymbol{y}$ and $\boldsymbol{z}=\boldsymbol{y}+\boldsymbol{\eta}$ ) about the streamwise direction. This is equivalent to the scalar forms (see paragraph at the bottom of p.7): $\left(\hat{R}_{i j k l} \hat{\eta}_{i} \hat{\eta}_{j} \hat{\eta}_{k} \hat{\eta}_{l}, \hat{R}_{4 j k l} \hat{\eta}_{j} \hat{\eta}_{k} \hat{\eta}_{l} \&\right.$ $\hat{R}_{4 j 4 l} \hat{\eta}_{j} \hat{\eta}_{l}$ ) remaining invariant to the $O(2)$ symmetry group about $\lambda=\boldsymbol{e}_{1}$ where $\hat{\eta}_{i}=\eta_{i} /|\boldsymbol{\eta}|$. Since App. (A.2) in [8] and App. A in [17] both show that the symmetry group can also be projected down to $\mathcal{S} O(2)$ sub-group without changing its representation of $\hat{R}_{\lambda j \mu l}$. (Note the error in Lie group designation on pp.132 \& 137 of ref. [8]). Since $\hat{R}_{\lambda j \mu l}\left(\boldsymbol{y}, \eta_{1}, \eta_{\perp}, \Delta \psi ; \tau_{0}\right)=\bar{R}_{\lambda j \mu l}\left(\boldsymbol{y}, \hat{\eta}_{1}, \hat{\eta}_{\perp} ; \tau_{0} ; \epsilon\right)$ for $\epsilon<1$ (see line below 18 , the latter tensor is also axi-symmetric. The temporal Fourier transformed tensor, $\bar{H}_{\lambda j \mu l}\left(\boldsymbol{y}, \hat{\eta}_{1}, \hat{\eta}_{\perp} ; \omega, \epsilon\right)$ in 4 is also, therefore, an axi-symmetric tensor as is its linear multiple, $\overline{\mathcal{H}}_{\lambda j \mu l}\left(\boldsymbol{y}, \hat{\eta}_{1}, \hat{\eta}_{\perp} ; \omega, \epsilon\right)$, defined below $(5)$.

We now define the spectral tensor, $\Phi_{\lambda j \mu l}(\boldsymbol{y}, \boldsymbol{k}, \omega)$,

$$
\Phi_{\lambda j \mu l}(\boldsymbol{y}, \boldsymbol{k}, \omega):=\int_{V(\boldsymbol{\eta})} \overline{\mathcal{H}}_{\lambda j \mu l}\left(\boldsymbol{y}, \hat{\eta}_{1}, \hat{\eta}_{\perp} ; \omega, \epsilon\right) e^{-i \boldsymbol{k} \cdot \boldsymbol{\eta}} d \boldsymbol{\eta}
$$

is equal to $(2 \pi)^{3}$ times the usual 3-dimensional spectrum defined by Eq. (2.4.3) on p.23 in Batchelor [18] and its complex conjugate enters the acoustic spectrum formula; see, for example, see formulae given by Eqs. (34) \& (36) in Afsar et al. [17]. The localized volume, $V(\boldsymbol{\eta})$ defined about the field point, $\boldsymbol{y}$ with triple integral over an elemental volume, $d \boldsymbol{\eta}$, defined in Cartesian co-ordinates by $d \boldsymbol{\eta}=\prod_{n=1}^{3} d \eta_{n}$. Inserting 13 shows that the $O(1)$ normalized correlation volume given by $d \boldsymbol{\eta}=d \boldsymbol{\eta} / l_{c}^{3}=\epsilon^{3} d \hat{\boldsymbol{\eta}}$ where $d \hat{\boldsymbol{\eta}}=O(1)$ in which the correlation length, $l_{c}$, possesses an upper bound, $l_{c} \sim D_{J}$; see paragraph below 14 . In cylindrical co-ordinates the normalized volume, $d \hat{\boldsymbol{\eta}}=\prod_{n=1}^{3} d \hat{\eta}_{n}=d \hat{\eta}_{1} \hat{\eta}_{\perp} d \hat{\eta}_{\perp} d \Delta \psi$ extends along a line in the streamwise direction of $O(1)$ length; a radius, $\eta_{\perp}$, of $O(\epsilon)$ extent and over a wedge, $\Delta \psi$, in the azimuthal direction of asymptotically small thickness that goes to zero like $o\left(\epsilon^{1 / 2}\right)$ (see ref.[10]).

From the definition of the Fourier transform, as a limit of a Fourier series (see Eqs. $7.3 \& 7.4$ on p.302 in Carrier et al. [19]), the integral over separation vector, $\boldsymbol{\eta}$, in (19) is defined over the continuum of wavenumbers, which in Cartesian co-ordinates is $\boldsymbol{k}=\left(k_{1}, \boldsymbol{k}_{\perp}\right)=\left(k_{1}, k_{2}, k_{3}\right)$. The theory of Fourier integrals expounded by Carrier $e$ t al. shows that the argument of the kernel of $(19)$ is such that $\boldsymbol{k} \cdot \boldsymbol{\eta}=O(1)$. . Hence, if we insert 13 in $\boldsymbol{k} \cdot \boldsymbol{\eta}=O(1)$ we obtain:

$$
\begin{aligned}
\boldsymbol{k} \cdot \boldsymbol{\eta}=O(1) & =\left(\delta_{i 1} \hat{\eta}_{1}+\delta_{i 2} \epsilon \hat{\eta}_{2}+\delta_{i 3} \epsilon^{2} \hat{\eta}_{3}\right) \hat{k}_{i} \\
& =\hat{k}_{1} \hat{\eta}_{1}+\left(\epsilon \gamma_{2}\right) \hat{k}_{2} \hat{\eta}_{2}+\left(\epsilon^{2} \gamma_{3}\right) \hat{k}_{3} \hat{\eta}_{3},
\end{aligned}
$$

where the normalized wavenumber vector is $\hat{\boldsymbol{k}}=l_{c} \boldsymbol{k}$. But since the $\left(\eta_{1}, \eta_{2}, \eta_{3}\right)$ integrals in 19 ) are independent, $(\boldsymbol{k} \cdot \boldsymbol{\eta})=O(1)$ requires that $\left(\epsilon \cdot \gamma_{2}\right)=O(1)$ and $\left(\epsilon^{2} \cdot \gamma_{3}\right)=O(1)$. The natural result is, therefore, that the $\boldsymbol{\eta}$-Fourier transform of the auto-covariance $55, \Phi_{\lambda j \mu l}(\boldsymbol{y}, \boldsymbol{k}, \omega)$, depends on transverse wavenumbers via gauge functions $\left(\gamma_{2}, \gamma_{3}\right)$ in 20 that scale like, $\gamma_{2} \sim 1 / \epsilon$ and $\gamma_{3} \sim 1 / \epsilon^{2}$. This implies that if $\hat{R}_{\lambda j \mu l}\left(\boldsymbol{y}, \eta_{1}, \eta_{2}, \eta_{3} ; \tau_{0}\right)$ depends on $\boldsymbol{\eta}$ through the strained separation vector in 13 , its spectrum, $\Phi_{\lambda j \mu l}(\boldsymbol{y}, \boldsymbol{k}, \omega)$, must depend on $\boldsymbol{k}$ via the following richest possible asymptotic scaling:

$$
\boldsymbol{k}:=\left\{\hat{k}_{1}, \epsilon^{-1} \hat{k}_{2}, \epsilon^{-2} \hat{k}_{3}\right\} l_{c}^{-1} \equiv\left(\delta_{i 1} \hat{k}_{1}+\delta_{i 2} \epsilon^{-1} \hat{k}_{2}+\delta_{i 3} \epsilon^{-2} \hat{k}_{3}\right) l_{c}^{-1},
$$

that is slowly-varying along co-ordinate directions in the transverse $(2-3)$ plane at arbitrary points in space/frequency $(\boldsymbol{y} ; \omega)=O(1)$ and where $\left(\hat{k}_{1}, \hat{k}_{2}, \hat{k}_{3}\right)=O(1)$. The dependence of $\Phi_{\lambda j \mu l}(\boldsymbol{y}, \boldsymbol{k}, \omega) \equiv \hat{\Phi}_{\lambda j \mu l}(\boldsymbol{y}, \hat{\boldsymbol{k}} ; \omega, \epsilon)$ on $\left(k_{2}, k_{3}\right)$ in 19) remains (at most) as large as $O\left(1 / \epsilon^{2}\right)$; anything less than this (i.e., the $O(1 / \epsilon)$ scaling in the normal $\hat{k}_{2}$ direction) is asymptotically smaller than this as $\epsilon \rightarrow 0$. The latter corresponds to the emergence of a distinct scale separation occurs for the physically-measured auto-covariance tensor (5) through the definition of the $\epsilon$ below (14). The magnitude of the slow-spectral decay of $\hat{\Phi}_{\lambda j \mu l}$ in the $\left(k_{2}, k_{3}\right)$ co-ordinates at any fixed spatial field point and frequency $(\boldsymbol{y} ; \omega)$ must, in a sense, correspond to a 'stretching mechanism' (being the linguistic antithesis of straining) — but this only results because of the aforementioned straining (i.e., spatial reduction) of the physical turbulence in the $\left(\eta_{2}, \eta_{3}\right)$ (or, $\left.\left(\eta_{\perp}, \Delta \psi\right)\right)$ spatial separations in 13 .

It is easy now to recover the Leib \& Goldstein result (Eqs. $39 \& 40$ in [21]) and (B.10) in [17] (see details in [10]). That is, $\hat{\Phi}_{\lambda j \mu l}^{*}(\boldsymbol{y}, \hat{\boldsymbol{k}} ; \omega, \epsilon)$ is proportional to the streamwise Fourier transform of the following Hankel transform (Eq.8.5.7 on p.962 in Morse \& Feshbach [22]):

$$
\hat{\Gamma}_{\lambda j \mu l}\left(\boldsymbol{y}, \hat{\eta}_{1}, \hat{k}_{\perp}^{m} ; \omega, \epsilon\right)=2 \pi \int_{0}^{\infty} \overline{\mathcal{H}}_{\lambda j \mu l}\left(\boldsymbol{y}, \hat{\eta}_{1}, \hat{\eta}_{\perp} ; \omega, \epsilon\right) J_{0}\left(\hat{k}_{\perp} \hat{\eta}_{\perp}\right) \hat{\eta}_{\perp} d \eta_{\perp},
$$

\footnotetext{
${ }^{\dagger}$ The limiting form of this defining property of Fourier transforms is exploited in the Abelian theorem as summarized on p. 36 of Noble [20]
} 
where the exponent, $m$, on the left side of 22 scaling the $\hat{k}_{\perp}$-dependency of the Hankel transform $\hat{\Gamma}_{\lambda j \mu l}\left(\boldsymbol{y}, \hat{\eta}_{1}, \hat{k}_{\perp}^{m} ; \omega, \epsilon\right)$ of the turbulence defined through the scripted tensor, $\overline{\mathcal{H}}_{\lambda j \mu l}$. This exponent is given by $m=2$ because the zeroth-order Bessel function, $J_{0}\left(\hat{k}_{\perp} \hat{\eta}_{\perp}\right)$, is an even function and therefore expands as even powers of its argument (Eq. 4.1 in Bowman [23]). Hence, the complex conjugate of (19] reduces to the single integral:

$$
\hat{\Phi}_{\lambda j \mu l}^{*}\left(\boldsymbol{y}, \hat{k}_{1}, \hat{k}_{\perp}^{2} ; \omega, \epsilon\right)=\int_{0}^{\infty} \hat{\Gamma}_{\lambda j \mu l}\left(\boldsymbol{y}, \hat{\eta}_{1}, \hat{k}_{\perp}^{2} ; \omega, \epsilon\right) e^{i k_{1} \eta_{1}} d \eta_{1}
$$

that is formally independent of the azimuthal separation, $\Delta \Psi$, in the transverse wavenumber $\left(k_{2}, k_{3}\right)$ space defined above. Whence, it follows from Lemma 3.2 in Afsar [8] that the left side of (23) can be replaced by its azimuthal average. Namely, $\hat{\Phi}_{\lambda j \mu l}^{*}\left(\boldsymbol{y}, \hat{k}_{1}, \hat{k}_{\perp}^{2} ; \omega, \epsilon\right) \equiv \bar{\Phi}_{\lambda j \mu l}^{*}\left(\boldsymbol{y}, \hat{k}_{1}, \hat{k}_{\perp}^{2} ; \omega, \epsilon\right)+o(1)$ up to an error that, commensurate with 17 , remains $o(1)$ to the order of magnitude of the analysis (in other words, as $\epsilon \rightarrow 0$ ). The azimuthal average of the spectral tensor 19 over a $\Delta \Psi\left(k_{2}, k_{3}\right)$ wedge is given by (3.18) in Ref. [8]. Moreover, the functional dependence of $\bar{\Phi}_{\lambda j \mu l}^{*}$ on $\hat{k}_{\perp}^{2}$ can obviously reduce to a dependence on $\hat{k}_{\perp}=\left|\boldsymbol{k}_{\perp}\right|$ since the latter maintains that Hankel transform $\hat{\Gamma}_{\lambda j \mu l}\left(\boldsymbol{y}, \hat{\eta}_{1}, \hat{k}_{\perp}^{m} ; \omega, \epsilon\right)$ in 22, , is an even function of $\boldsymbol{k}_{\perp}$ when inserted into the remaining axial transform, 23.

\section{E. Asymptotic structure and expansion of tensors $\bar{\Phi}_{\lambda j \mu l}^{*}$ and $\bar{R}_{\lambda j \mu l}$ with next-order corrections}

Since the square magnitude of the wavenumber vector $k_{i}=l_{c}^{-1} \hat{k}_{i}$ is given by $\hat{k}_{i}^{2}=\sum_{n=1}^{3} \epsilon^{-2(n-1)} \hat{k}_{n}^{2}$ after using 21 , we easily find that the square of its transverse components then scale like $k_{\perp}^{2}=\left(\gamma_{2}^{2} \hat{k}_{2}^{2}+\gamma_{3}^{2} \hat{k}_{3}^{2}\right) l_{c}^{-2}$ or $\hat{k}_{\perp}^{2}=\left(\epsilon^{-2} \hat{k}_{2}^{2}+\epsilon^{-4} \hat{k}_{3}^{2}\right)$ via [20] \& 21]. Inserting this into Eq. (A.6) of Afsar [8] (when the principal direction is $\lambda=\boldsymbol{e}_{1}$ ) then shows that the spectral tensor $\bar{\Phi}_{\lambda j \mu l}^{*}\left(\boldsymbol{y}, \hat{k}_{1}, \hat{k}_{\perp}^{2} ; \omega, \epsilon\right)$ expands increasing powers of gauge functions $\left(\gamma_{2}, \gamma_{3}\right):=\left(\epsilon^{-1}, \epsilon^{-2}\right)$ in 20 ; i.e.,

$$
\begin{aligned}
\bar{\Phi}_{\lambda j \mu l}^{*}\left(\boldsymbol{y}, \hat{k}_{1}, \hat{k}_{\perp}^{2} ; \omega, \epsilon\right) & =\bar{\Phi}_{\lambda j \mu l}^{*(1)}\left(\boldsymbol{y}, \hat{k}_{1}, 0 ; \omega, \epsilon\right) \\
& +\bar{\Phi}_{\lambda j \mu l}^{*(2)}\left(\boldsymbol{y}, \hat{k}_{1}, \hat{k}_{\perp}^{2}\left(\hat{k}_{2} ; \gamma_{2}\right) ; \omega, \epsilon\right) \\
& +\bar{\Phi}_{\lambda j \mu l}^{*(3)}\left(\boldsymbol{y}, \hat{k}_{1}, \hat{k}_{\perp}^{2}\left(\hat{k}_{2}, \hat{k}_{3} ; \gamma_{2}, \gamma_{3}\right) ; \omega, \epsilon\right) .
\end{aligned}
$$

This result can be written much more succinctly as the following sum of sub-spectral tensors: $\bar{\Phi}_{\lambda j \mu l}^{*}\left(\boldsymbol{y}, \hat{k}_{1}, \hat{k}_{\perp}^{2} ; \omega, \epsilon\right)=$ $\sum_{n=1}^{3} \bar{\Phi}_{\lambda j \mu l}^{*(n)}\left(\boldsymbol{y}, \hat{k}_{1},\left(\hat{k}_{\perp}^{2}\right)^{(n)} ; \omega, \epsilon\right)$. The third argument denotes $\left(\hat{k}_{\perp}^{2}\right)^{(1)}=\gamma^{*}\left(\hat{k}_{\perp}^{2}\right)^{(2)}=\hat{k}_{\perp}^{2}\left(\hat{k}_{2} ; \gamma_{2}\right)=\gamma_{2}^{2} \hat{k}_{2}^{2}+O\left(\gamma_{3}^{2}\right)$ and $\left(\hat{k}_{\perp}^{2}\right)^{(3)}=\hat{k}_{\perp}^{2}\left(\hat{k}_{2}, \hat{k}_{3} ; \gamma_{2}, \gamma_{3}\right)$. The latter two arguments of the sub-spectral tensors are found using the expansion for $k_{\perp}^{2}$ above 24 , where $\left(\hat{k}_{\perp}^{2}\right)^{(3)}$ includes both $O\left(\gamma_{2}^{2}\right)$ and $O\left(\gamma_{3}^{2}\right)$ contributions. But since the asymptotic expansion for the Fourier-type integrals (p.256 of Carrier et al. [19]) shows that large (infinite) transverse wave-number, $\hat{k}_{\perp}^{2} \rightarrow \infty$ corresponds to small $\hat{\eta}_{\perp}=\left|\hat{\eta}_{\perp}\right| \rightarrow 0$; i.e., infinitesimally small transverse correlation length. Therefore, retaining only terms $O\left(\gamma_{2}\right) \equiv O(1 / \epsilon)$ in the sub-spectral tensor $\bar{\Phi}_{\lambda j \mu l}^{*(2)}\left(y, \hat{k}_{1}, \hat{k}_{\perp}^{2}\left(\hat{k}_{2} ; \gamma_{2}\right) ; \omega, \epsilon\right)$ since the P\&M/HB turbulence data indicates that the transverse $\eta_{2}$ correlation length, although small, is non-zero, commensurate with the small but non-zero value of the $\epsilon$-parameter. The $O\left(\gamma_{2}\right)$ retention corresponds to only keeping terms linear in $k_{2}$ in the complete axi-symmetric expansion formula, (A.6) in Afsar [8]). Inasmuch as $\bar{\Phi}_{\lambda j \mu l}^{*(2)}\left(\boldsymbol{y}, \hat{k}_{1}, \hat{k}_{\perp}^{2}\left(\hat{k}_{2} ; \gamma_{2}\right) ; \omega, \epsilon\right)$ depends on $\hat{k}_{\perp}^{2}\left(\hat{k}_{2} ; \gamma_{2}\right)$ via $\hat{k}_{\perp}=\left|\hat{k}_{\perp}\right|=\gamma_{2}\left|\hat{k}_{2}\right|+O\left(\gamma_{3}^{2}\right) \mid$. Eq. (A.6) in [8] still shows that even this retention to lowest order in $\gamma_{2}$ in the scaling for $\hat{k}_{\perp}^{2}\left(\hat{k}_{2} ; \gamma_{2}\right)$ produces a permutative expansion as a power series in increasing algebraic powers of $\hat{k}_{2}$ of the form $\sum_{r} \hat{\alpha}_{r} \hat{k}_{2}^{r}$ where the index $r$ runs between the integral range, $r \in[1,4]$ and $\hat{\alpha}_{r}$ is scalar function of $\left(y, k_{1}, k_{\perp}^{2}, \omega\right)$. Higher powers of $\gamma_{2}$ in (A.6) of ref. [8] will tend to infinity faster like $O\left(1 / \epsilon^{r}\right)$ (where $\left.r>1\right)$ by [20] \& 21. Slow spectral variation of $\bar{\Phi}_{\lambda j \mu l}^{*(2)}$ (and therefore $\bar{\Phi}_{\lambda j \mu l}^{*}$ ) in transverse wavenumber space of this type will result in the physical space tensor $\bar{R}_{\lambda j \mu l}$ to immediately de-correlate with $\hat{\eta}_{\perp}([\overline{19]} \&[20])$ or have an amplitude that is much less than unity since such $O\left(\gamma_{2}^{r>1}\right)$ terms equate to components of the physical space auto-covariance tensor (Eq. A.10 and p.137 in [8]) that are of very small magnitude; see, for example, [24].

\footnotetext{
† This corresponds to the fully ' $\boldsymbol{k}_{\perp}$-independent’ Afsar result, Eq. (A.11) in ref. [8].
} 
These arguments imply that $\bar{\Phi}_{\lambda j \mu l}^{*(2)}\left(\boldsymbol{y}, \hat{k}_{1}, \hat{k}_{\perp}^{2}\left(\hat{k}_{2} ; \gamma_{2}\right) ; \omega, \epsilon\right)=O\left(\gamma_{2}\right)+O\left(\gamma_{2}^{2}\right)+\ldots+O\left(\gamma_{2}^{4}\right)$. In [10] we show that the $O\left(\gamma_{2}\right)$-truncated form of the sub-spectral tensor $\bar{\Phi}_{\lambda j \mu l}^{*(2)}=\bar{\Phi}_{i j k l}^{*(2)}\left(\boldsymbol{y}, \hat{k}_{1}, \gamma_{2}\left|\hat{k}_{2}\right| ; \omega, \epsilon\right)$ in 24 can be expressed in the following form for the $(\lambda, \mu)=(i, k)$ momentum-flux associated tensor:

$$
\begin{aligned}
\bar{\Phi}_{i j k l}^{*(2)}\left(\boldsymbol{y}, \hat{k}_{1}, \gamma_{2}\left|\hat{k}_{2}\right| ; \omega, \epsilon\right) & =\left[\delta_{i j}\left(\delta_{l 1} \delta_{k 2}+\delta_{k 1} \delta_{l 2}\right)+\delta_{k l}\left(\delta_{i 2} \delta_{j 1}+\delta_{j 2} \delta_{i 1}\right)\right] \bar{A}_{5}\left(\boldsymbol{y}, \hat{k}_{1}, \gamma_{2}\left|\hat{k}_{2}\right| ; \omega, \epsilon\right) \\
& +\left[\delta_{i k}\left(\delta_{l 1} \delta_{j 2}+\delta_{j 1} \delta_{l 2}\right)+\delta_{j l}\left(\delta_{i 2} \delta_{k 1}+\delta_{k 2} \delta_{i 1}\right)\right. \\
& \left.+\delta_{i l}\left(\delta_{j 2} \delta_{k 1}+\delta_{k 2} \delta_{j 1}\right)+\delta_{j k}\left(\delta_{l 1} \delta_{i 2}+\delta_{i 1} \delta_{l 2}\right)\right] \bar{A}_{8}\left(\boldsymbol{y}, \hat{k}_{1}, \gamma_{2}\left|\hat{k}_{2}\right| ; \omega, \epsilon\right) \\
& +\left[\delta_{i 1} \delta_{j 1}\left(\delta_{l 1} \delta_{k 2}+\delta_{k 1} \delta_{l 2}\right)+\delta_{k 1} \delta_{l 1}\left(\delta_{i 1} \delta_{j 2}+\delta_{i 2} \delta_{j 1}\right)\right] \bar{A}_{10}\left(\boldsymbol{y}, \hat{k}_{1}, \gamma_{2}\left|\hat{k}_{2}\right| ; \omega, \epsilon\right)+O\left(\gamma_{2}^{2}\right) .
\end{aligned}
$$

Eqs. (19), (23), (22), (4) and the linear relation below it now show that each term in (24) is defined by a physical-space auto-covariance that has the same tensorial structure. This immediately suggests that, commensurate with [24], $\bar{R}_{\lambda j \mu l}\left(\boldsymbol{y}, \hat{\eta}_{1}, \hat{\eta}_{\perp} ; \omega, \epsilon\right):=\sum_{n=1}^{3} \bar{R}_{\lambda j \mu l}^{(n)}\left(\boldsymbol{y}, \hat{\eta}_{1}, \hat{\eta}_{\perp}\left(\delta_{n 2} \hat{\eta}_{2}, \delta_{n 3} \hat{\eta}_{3}\right) ; \omega, \epsilon\right)$ where $\left(\delta_{n 2}, \delta_{n 3}\right)$ are unit tensors. In full form this takes the form:

$$
\bar{R}_{\lambda j \mu l}\left(\boldsymbol{y}, \hat{\eta}_{1}, \hat{\eta}_{\perp} ; \omega, \epsilon\right):=\bar{R}_{\lambda j \mu l}^{(1)}\left(\boldsymbol{y}, \hat{\eta}_{1}, 0 ; \omega, \epsilon\right)+\bar{R}_{\lambda j \mu l}^{(2)}\left(\boldsymbol{y}, \hat{\eta}_{1}, \hat{\eta}_{\perp}\left(\hat{\eta}_{2}\right) ; \omega, \epsilon\right)+\bar{R}_{\lambda j \mu l}^{(3)}\left(\boldsymbol{y}, \hat{\eta}_{1}, \hat{\eta}_{\perp}\left(\hat{\eta}_{2}, \hat{\eta}_{3}\right) ; \omega, \epsilon\right)
$$

where $\bar{R}_{i j k l}^{(1)}\left(\boldsymbol{y}, \hat{\eta}_{1}, 0 ; \omega, \epsilon\right)$ is given by $\sqrt{6}$ for the momentum flux $(\lambda, \mu)=(i, k)$ components and using $[25], \bar{R}_{i j k l}^{(2)}$, at lowest order in $\epsilon$, expands as:

$$
\begin{aligned}
\bar{R}_{i j k l}^{(2)}\left(\boldsymbol{y}, \hat{\eta}_{1}, \hat{\eta}_{\perp}\left(\hat{\eta}_{2}\right) ; \omega, \epsilon\right) & =\left[\delta_{i j}\left(\delta_{l 1} \delta_{k 2}+\delta_{k 1} \delta_{l 2}\right)+\delta_{k l}\left(\delta_{i 2} \delta_{j 1}+\delta_{j 2} \delta_{i 1}\right)\right] \bar{R}_{3312}\left(\boldsymbol{y}, \hat{\eta}_{1}, \hat{\eta}_{\perp}\left(\hat{\eta}_{2}\right) ; \omega, \epsilon\right) \\
& +\left[\delta_{i k}\left(\delta_{l 1} \delta_{j 2}+\delta_{j 1} \delta_{l 2}\right)+\delta_{j l}\left(\delta_{i 2} \delta_{k 1}+\delta_{k 2} \delta_{i 1}\right)\right. \\
& \left.+\delta_{i l}\left(\delta_{j 2} \delta_{k 1}+\delta_{k 2} \delta_{j 1}\right)+\delta_{j k}\left(\delta_{l 1} \delta_{i 2}+\delta_{i 1} \delta_{l 2}\right)\right] \bar{R}_{3231}\left(\boldsymbol{y}, \hat{\eta}_{1}, \hat{\eta}_{\perp}\left(\hat{\eta}_{2}\right) ; \omega, \epsilon\right) \\
& +\left[\delta_{i 1} \delta_{j 1}\left(\delta_{l 1} \delta_{k 2}+\delta_{k 1} \delta_{l 2}\right)+\right. \\
& \left.+\delta_{k 1} \delta_{l 1}\left(\delta_{i 1} \delta_{j 2}+\delta_{i 2} \delta_{j 1}\right)\right]\left(\bar{R}_{1112}-\bar{R}_{3312}-2 \bar{R}_{3231}\right)\left(\boldsymbol{y}, \hat{\eta}_{1}, \hat{\eta}_{\perp}\left(\hat{\eta}_{2}\right) ; \omega, \epsilon\right)+O\left(\epsilon^{2}\right),
\end{aligned}
$$

to within an $O\left(\epsilon^{2}\right)$ remainder and, therefore, generalizes Eq. (A.11) in [8] to include the $O(\epsilon)$ correction for a turbulence field within the jet that depends on the transverse separation with magnitude that expands as $\hat{\eta}_{\perp}=\left|\hat{\eta}_{\perp}\right|=$ $\epsilon \hat{\eta}_{2}+O\left(\epsilon^{2}\right)$. In other words, the first two terms of the functional decomposition in 26) can be re-arranged to read, $\bar{R}_{\lambda j \mu l}\left(\boldsymbol{y}, \hat{\eta}_{1}, \hat{\eta}_{\perp} ; \omega, \epsilon\right)-\bar{R}_{\lambda j \mu l}^{(1)}\left(\boldsymbol{y}, \hat{\eta}_{1}, 0 ; \omega, \epsilon\right)=$ Eq. $(27)+o(\epsilon)$ where $\bar{R}_{\lambda j \mu l}^{(1)}$ is given by 6 . This then provides a two-term asymptotic expansion formula for $\bar{R}_{\lambda j \mu l}$ which we validate next using LES data for the momentum flux components $(\lambda, \mu)=(i, k)$.

IV. Using LES data to validate the 2-term asymptotic expansion formulae (26) \& (27)

The quartilinear tensor form (or, square norm) of $R_{i j k l}$ can now be re-computed using 26$] \&(27)$. That is,

$$
\begin{aligned}
{\left[\frac{R_{i j k l} R_{i j k l}}{R_{1111}^{2}}\right](y, 0 ; 0) } & =1+\frac{2 R_{2222}^{2}}{R_{1111}^{2}}(y, 0 ; 0)+4 \cdot\left[\frac{R_{1122}^{2}+\frac{1}{2} R_{2233}^{2}}{R_{1111}^{2}}\right](y, 0 ; 0)+8 \cdot\left[\frac{R_{1212}^{2}+\frac{1}{2} R_{2323}^{2}}{R_{111}^{2}}\right](y, 0 ; 0) \\
& +\quad+12 \frac{R_{3312}^{2}}{R_{1111}^{2}}+32 \frac{R_{3231}^{2}}{R_{1111}^{2}}+4 \cdot\left[\frac{\left(R_{1112}-R_{3312}-2 R_{3231}\right)^{2}}{R_{1111}^{2}}\right](y, 0 ; 0)+16 \frac{R_{3312} R_{3231}}{R_{1111}^{2}}(y, 0 ; 0) \\
& +8 \cdot\left[\frac{R_{3312}\left(R_{1112}-R_{3312}-2 R_{3231}\right)}{R_{1111}^{2}}\right](y, 0 ; 0)+16 \cdot\left[\frac{R_{3231}\left(R_{1112}-R_{3312}-2 R_{3231}\right)}{R_{1111}^{2}}\right](y, 0 ; 0)
\end{aligned}
$$

Figs. $107-\left(12\right.$ show much closer agreement between the direct LES formula for the square-norm $R_{i j k l}^{2} / R_{1111}^{2}$ in 9$\}$ and 28) compared to the previous calculation of (11) that represents the leading term in the general asymptotic formula, 

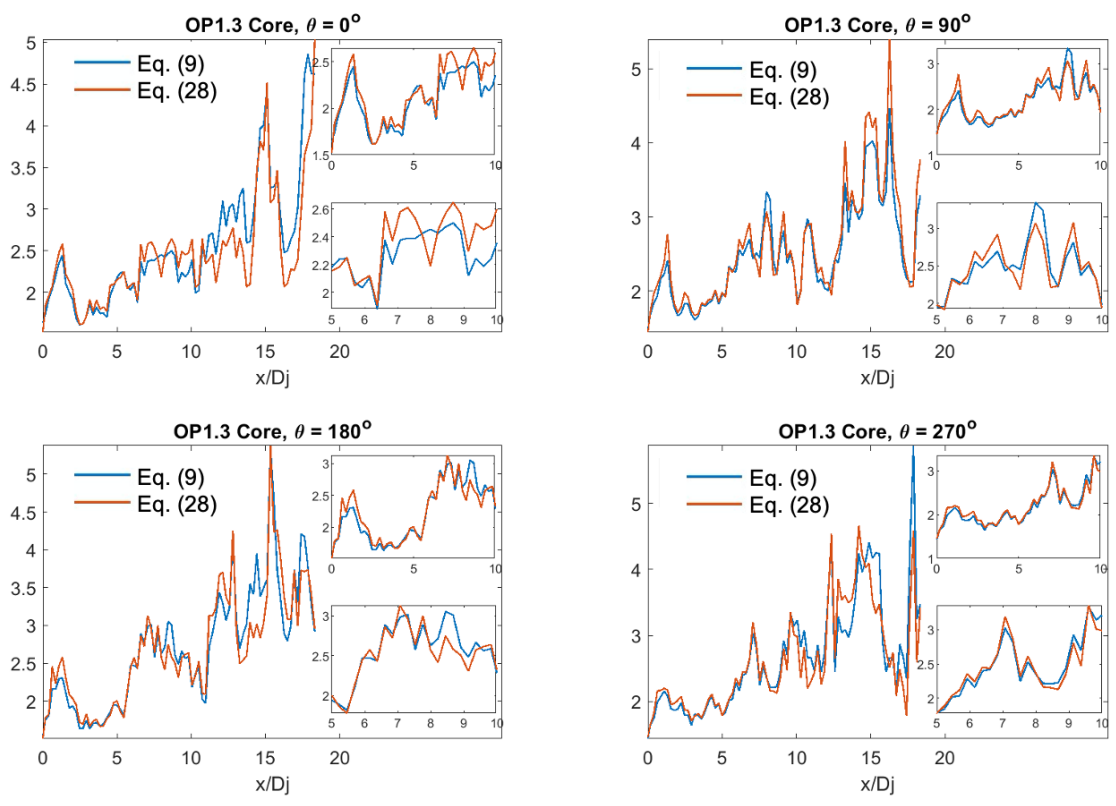

Fig. 10 Axial variation of square norm $R_{i j k l}^{2} / R_{1111}^{2}$ given by Eqs. (9) (LES data - no approximation) and (28) (LES data - axi-symmetry given by Eqs. 6, 26, 27) for OP1.3 at the core shear layer radial location, $r / D_{J}=0.18$.

(26). The results in Figs. (9)-(12) indicate a difference of less than $1 \%$ between Eqs. (9) and (28) at the locations at almost all locations from the nozzle exit to the end of potential core. This agreement is closer in the core radius for OP1.3 compared to the bypass radius for both OPs. However, here too, the difference is considerably better than in Figs. (1)-33. But the LES data (in Figs. $13-21$ also shows that the components $\bar{R}_{3312}$ and $\bar{R}_{3231}$ are largely negligible (i.e. $\bar{R}_{3312}, \bar{R}_{3231} \ll 1$ at the core radius and bypass radius locations $\left(r / D_{j}=0.18\right.$ and $r / D_{j}=0.38$ respectively) where the turbulence kinetic energy is largest. The component $R_{1112}$ appears to retain a significant amount of turbulence energy relative to $R_{1111}$ this is evidently owing to the fact that 3 of tensor suffixes are contracted in the axial direction for this component. The bar charts in Figs. $16-21$ s show that the amplitude ratio $R_{1112} / R_{1111}$ remains bounded between 0.15 and almost 0.3 for both operating points at streamwise locations at the start and end of the potential core with an almost uniformity across the azimuthal planes - particularly at the streamwise locations closer to the start of the potential core (i.e., at $\sim 6 D_{j}$ ). This is consistent with the numerical results of Karabasov et al.[25].

Hence, using this information, the sub-tensor $\bar{R}_{i j k l}^{(2)}\left(\boldsymbol{y}, \hat{\eta}_{1}, \hat{\eta}_{\perp}\left(\hat{\eta}_{1}, \hat{\eta}_{2}\right) ; \omega, \epsilon\right)$ in 27 can be justifiably approximated to the following:

$\begin{aligned} \bar{R}_{i j k l}^{(2)}\left(\boldsymbol{y}, \hat{\eta}_{1}, \hat{\eta}_{\perp}\left(\hat{\eta}_{1}, \hat{\eta}_{2}\right) ; \omega, \epsilon\right) & \rightarrow\left[\delta_{i 1} \delta_{j 1}\left(\delta_{l 1} \delta_{k 2}+\delta_{k 1} \delta_{l 2}\right)\right. \\ + & \left.\delta_{k 1} \delta_{l 1}\left(\delta_{i 1} \delta_{j 2}+\delta_{i 2} \delta_{j 1}\right)\right]\left(\bar{R}_{1112}-\bar{R}_{3312}-2 \bar{R}_{3231}\right)\left(\boldsymbol{y}, \hat{\eta}_{1}, \hat{\eta}_{\perp}\left(\hat{\eta}_{1}, \hat{\eta}_{2}\right) ; \omega, \epsilon\right)+O\left(\epsilon^{2}\right),\end{aligned}$

since the difference in small $O(\epsilon) \sim\left(\bar{R}_{1112}, \bar{R}_{3312}, \bar{R}_{3231}\right)$ quantities for the red coloured terms in $(29):\left(\bar{R}_{1112}-\bar{R}_{3312}-2 \bar{R}_{3231}\right)$ could potentially be larger than the small quantities $\left(\bar{R}_{3312}, \bar{R}_{3231}\right)$ themselves due to the fact that 'almost-streamwise' term $\bar{R}_{1112}$ is significantly larger than the latter auto-covariance components. Our LES data (discussed below) confirms this. The natural follow-on to this approximation is that the momentum flux $(\lambda, \mu)=(i, k)$ components of the asymptotic expansion in 26) can be commensurately approximated to the following form:

$$
\begin{aligned}
\bar{R}_{i j k l}\left(\boldsymbol{y}, \hat{\eta}_{1}, \hat{\eta}_{\perp} ; \omega, \epsilon\right) & =\bar{R}_{i j k l}^{(1)}\left(\boldsymbol{y}, \hat{\eta}_{1}, 0 ; \omega, \epsilon\right) \\
& +\left[\delta_{i 1} \delta_{j 1}\left(\delta_{l 1} \delta_{k 2}+\delta_{k 1} \delta_{l 2}\right)\right. \\
& \left.+\delta_{k 1} \delta_{l 1}\left(\delta_{i 1} \delta_{j 2}+\delta_{i 2} \delta_{j 1}\right)\right]\left(\bar{R}_{1112}-\bar{R}_{3312}-2 \bar{R}_{3231}\right)\left(\boldsymbol{y}, \hat{\eta}_{1}, \hat{\eta}_{\perp}\left(\hat{\eta}_{1}, \hat{\eta}_{2}\right) ; \omega, \epsilon\right)+o(\epsilon)
\end{aligned}
$$



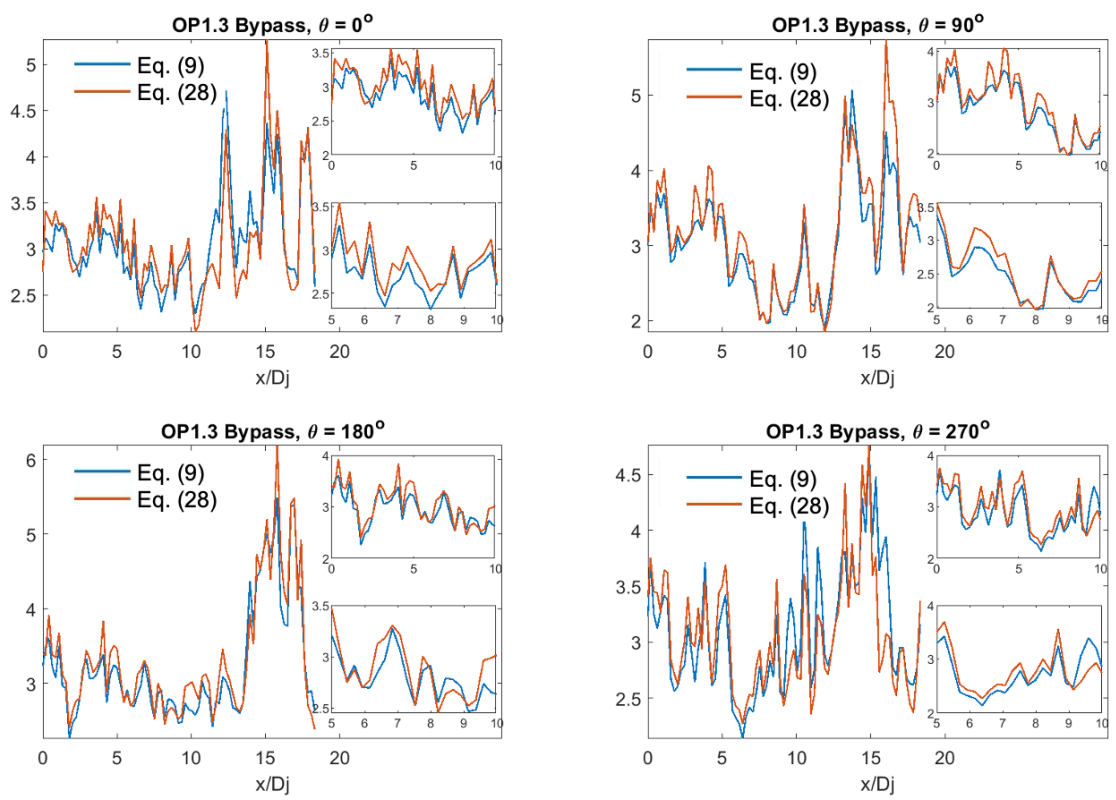

Fig. 11 Axial variation of square norm $R_{i j k l}^{2} / R_{111}^{2}$ given by Eqs. (9) (LES data - no approximation) and (28) (LES data - axi-symmetry given by Eqs. 6, 26, 27) for OP1.3 at the bypass shear layer radial location, $r / D_{J}=0.38$.

where $\bar{R}_{i j k l}^{(1)}\left(\boldsymbol{y}, \hat{\eta}_{1}, 0 ; \omega, \epsilon\right)$ is again given by $\sqrt{6}$ for the momentum flux components, 30 . Finally, the square norm 28 may be approximated to retain only the term linear in the difference $\left|R_{i j k l}\right| \equiv R_{1111} \sqrt{R_{i j k l}^{2} / R_{1111}^{2}} \sim$ $\left(\bar{R}_{1112}-R_{3312}-2 \bar{R}_{3231}\right)$ :

$$
\begin{aligned}
{\left[\frac{R_{i j k l} R_{i j k l}}{R_{1111}^{2}}\right](y, 0 ; 0) } & \rightarrow 1+\frac{2 R_{2222}^{2}}{R_{1111}^{2}}(\boldsymbol{y}, 0 ; 0)+4 \cdot\left[\frac{R_{1122}^{2}+\frac{1}{2} R_{2233}^{2}}{R_{1111}^{2}}\right](y, 0 ; 0)+8 \cdot\left[\frac{R_{1212}^{2}+\frac{1}{2} R_{2323}^{2}}{R_{1111}^{2}}\right](y, 0 ; 0) \\
& +4 \cdot\left[\frac{\left(R_{1112}-R_{3312}-2 R_{3231}\right)^{2}}{R_{1111}^{2}}\right](\boldsymbol{y}, 0 ; 0)
\end{aligned}
$$

to within an $O\left(\epsilon^{2}\right)$ remainder term. We can make one further approximation now, which is to retain only the $\bar{R}_{1112}$ term in the pre-factor for the term in red. That is,

$$
\begin{aligned}
{\left[\frac{R_{i j k l} R_{i j k l}}{R_{1111}^{2}}\right](y, 0 ; 0) } & \rightarrow 1+\frac{2 R_{2222}^{2}}{R_{1111}^{2}}(\boldsymbol{y}, 0 ; 0)+4 \cdot\left[\frac{R_{1122}^{2}+\frac{1}{2} R_{2233}^{2}}{R_{1111}^{2}}\right](y, 0 ; 0)+8 \cdot\left[\frac{R_{1212}^{2}+\frac{1}{2} R_{2323}^{2}}{R_{1111}^{2}}\right](\boldsymbol{y}, 0 ; 0) \\
& +4 \cdot\left[\frac{R_{1112}^{2}}{R_{1111}^{2}}\right](\boldsymbol{y}, 0 ; 0)
\end{aligned}
$$

In other words, the square-norm of the auto-covariance tensor, $R_{i j k l}$ in $(32)$ is equal to the sum of (11) (given by the leading term in the expansion formula, Eq. 26 for $(\mu, \lambda=i, k)$ only) and the extra contribution from the 'almost-streamwise' term, $\bar{R}_{1112}$ in form of $\left(2 \bar{R}_{1222} / \bar{R}_{1111}\right)^{2}$.

In Figs. 222 24, we show that the amplitude pre-factor $\left(\bar{R}_{1112}-\bar{R}_{3312}-2 \bar{R}_{3231}\right)$ in 30 (and its analytic consequence 31) remains of the order of $(0.1-0.2)$ when normalized by $R_{1111}$ (Figs. 22 24) or $(0.4-0.6)$ when normalized by $R_{1212}$ (the auto-covariance component that is dominant for the peak noise radiation; see [7] \& [9]). This is shown in Fig. 25. 

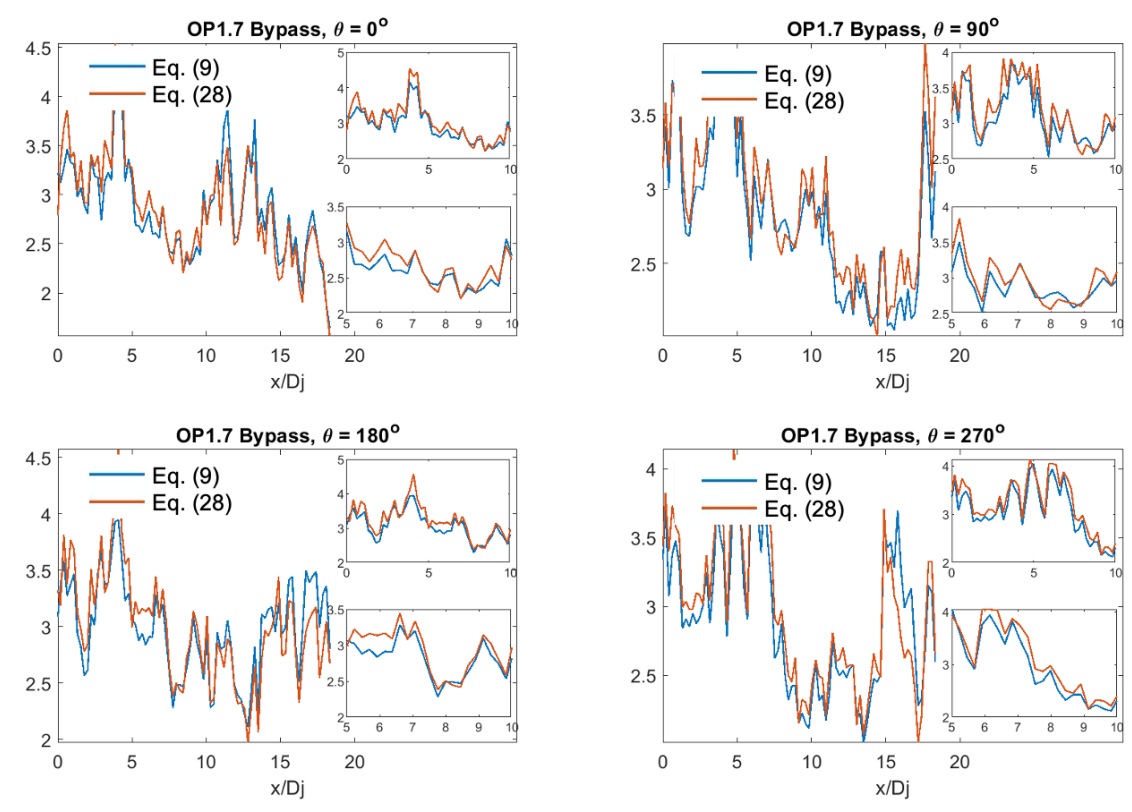

Fig. 12 Axial variation of square norm $R_{i j k}^{2} / R_{u 11}^{2}$ given by Eqs. (9) (LES data - no approximation) and (28) (LES data - axi-symmetry given by Eqs. 6, 26, 27) for OP1.7 at the bypass shear layer radial location, $r / D_{J}=0.38$.

\section{Discussion and Conclusions}

On p. 241 of their paper, Pokora \& McGuirk [11] wrote that: "These azimuthal correlations (i.e., $R_{11}$ and $R_{1111}$ ) are significantly flatter than the axial or radial correlations for quite small increases in the separation vector". The smallness of this variation has analytical consequences for the Reynolds stress auto-covariance tensor $\left(R_{i j k l}\right)$ in round jet flows. We have shown that it naturally implies that the latter tensor depends on a strained separation vector with richest asymptotic balance between transverse components 2 and 3 given by Eq. (13). Extending this definition to wave-number spectral space for the spatial Fourier transform of $R_{i j k l}$ in 21 allows an extended 2-term asymptotic formula for the auto-covariance tensor to be developed. This is given by our Eqs. 26] \& 27]. The latter formulae when expressed by the square-norm (i.e. the quartilinear tensor form) in Eq.28) compares very favorably with LES data for complex jet flow fields of our interest in this paper. Indeed the extended the theory of axisymmetric turbulence developed herein recovers the numerical results uncovered by Karabasov et al. [25], namely that the auto-covariance tensor component $R_{1112}$ remains sizeable at the end of the potential core region where the turbulence kinetic energy is large. Future work will aim to explore the impact of this term in acoustic analogy models of jet noise.

\section{Acknowledgments}

MZA would like to thank Strathclyde University for financial support from the Chancellor's Fellowship. The LES data for this work was performed in a previous project that was been supported by the Aero Acoustic Research Consortium and the Engineering and Physical Sciences Research Council (EP/S002065/1). The authors gratefully acknowledge their support.

\section{References}

[1] Afsar, M. Z., Gryazev, V., Markesteijn, A. P., and Karabasov, S. A., "Effect of large-scale mixing on the axisymmetric structure of turbulence correlations in complex dual-stream jets," 26th AIAA Aviation Conference, 2020.

[2] Gryazev, V., Markesteijn, A. P., and Karabasov, S. A., "Low-Order models of dual-stream jet noise with temperature effects based on the Goldstein generalised acoustic analogy," 25th AIAA Aeroacoustics Conference, 2019. 

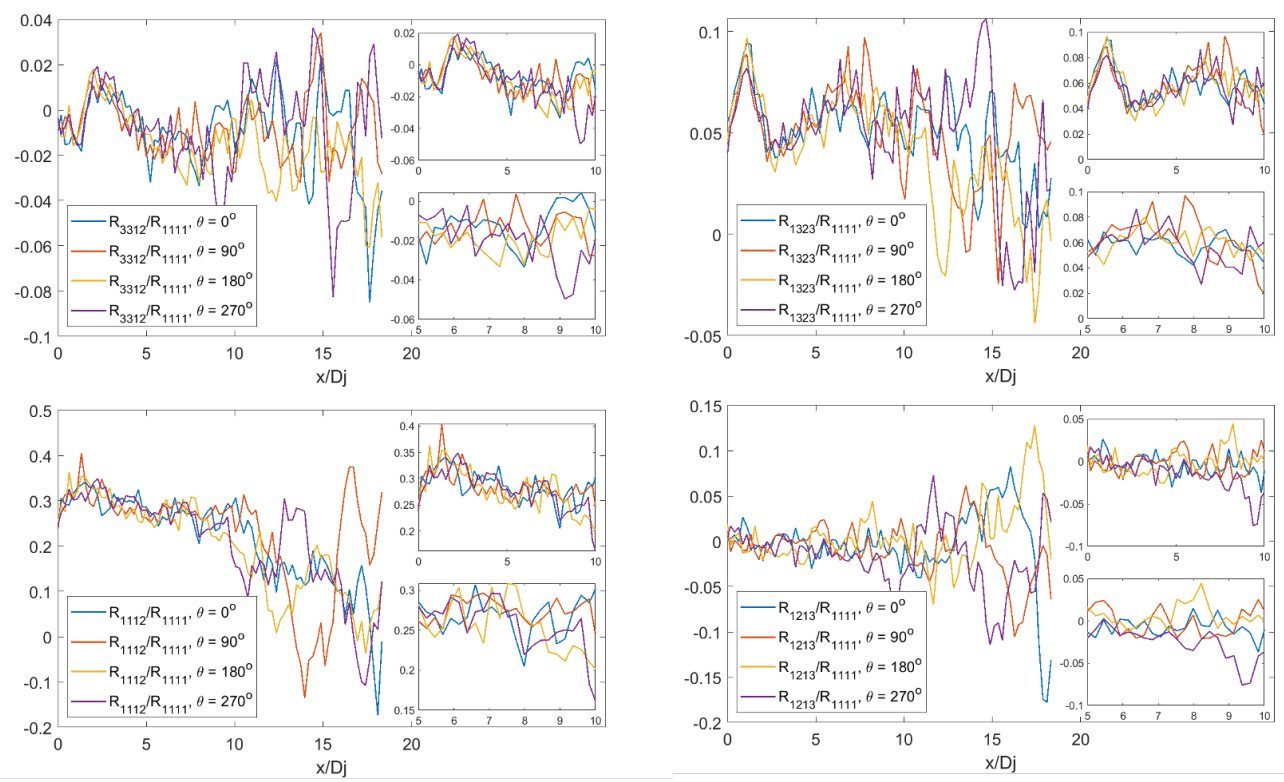

Fig. 13 Axial variation of extra components $\left(R_{3312}, R_{1323}, R_{1112}, R_{1213}\right.$ at 4 azimuthal planes: $\psi=$ $\left(0^{\circ}, 90^{\circ}, 180^{\circ}, 270^{\circ}\right)$ for $\mathrm{OP} 1.3$ at the core shear layer radial locations $r / D_{j}=0.18$ respectively.

[3] Semiletov, V. A., Karabasov, S. A., and Markesteijn, A. P., "Empiricism-free noise calculation from LES solution based on Goldstein generalized acoustic analogy: volume noise sources and mean flow effects," 21st AIAA/CEAS Aeroacoustics Conference, 2015.

[4] Goldstein, M. E., “A generalized acoustic analogy,” J. Fluid Mech., Vol. 488, 2003, p. 315.

[5] Afsar, M. Z., Sescu, A., and Sassanis, V. G., "Effect of non-parallel mean flow on the acoustic spectrum of heated supersonic jets: Explanation of "jet quietening”," Phys. Fluids, Vol. 31, 105107, 2019.

[6] Afsar, M. Z., Goldstein, M. E., and Fagan, A. M., "Enthalpy flux/Momentum flux coupling in the acoustic spectrum of heated jets," AIAA J., Vol. 49, 2011, p. 2522.

[7] Afsar, M. Z., "Asymptotic properties of the overall sound pressure level of sub-sonic air jets using isotropy as a paradigm," $J$. Fluid Mech., Vol. 664, 2010, p. 510.

[8] Afsar, M. Z., "Insight into the two-source structure of jet noise using a generalized shell model of turbulence," Euro. J. Mech. B/Fluids, Vol. 31, 2012, pp. 129-139.

[9] Karabasov, S. A., Afsar, M. Z., Hynes, T. P., Dowling, A. P., McMullan, W. A., Pokora, C. D., Page, G. J., and McGuirk, J. J., "Jet noise: acoustic analogy informed by Large-Eddy Simulation," AIAA J., Vol. 48, 2010, p. 1312.

[10] Afsar, M. Z., Gryazev, V., Markesteijn, A. P., and Karabasov, S. A., "Effect of large-scale mixing induced by complex flow fields on the axisymmetric representation of high-order turbulence correlation functions," In prep. for J. Fluid Mech., 2021.

[11] Pokora, C. D., and McGuirk, J. J., "Stereo-PIV measurements of spatio-temporal turbulence correlations in an axisymmetric jet," J. Fluid Mech., Vol. 778, 2015, p. 216.

[12] Harper-Bourne, M., "Jet noise turbulence measurements," 9th AIAA/CEAS Aeroacoustics Conference, Hilton Head, SC, USA., 2003.

[13] Davies, P. O. A. L., Fisher, M. J., and Barratt, M. J., “The characteristics of turbulence in the mixing region of a round jet," J. Fluid Mech., Vol. 15, 1962, p. 337.

[14] Morris, P. J., and Zaman, K., "Velocity measurements in jets with application to noise source modeling," J. Sound \& Vib., Vol. 329, 2010, p. 394.

[15] Gradshetyn, I. S., and Ryzhik, I. M., Tables of Integrals, Series and Products, chapter and pages. 

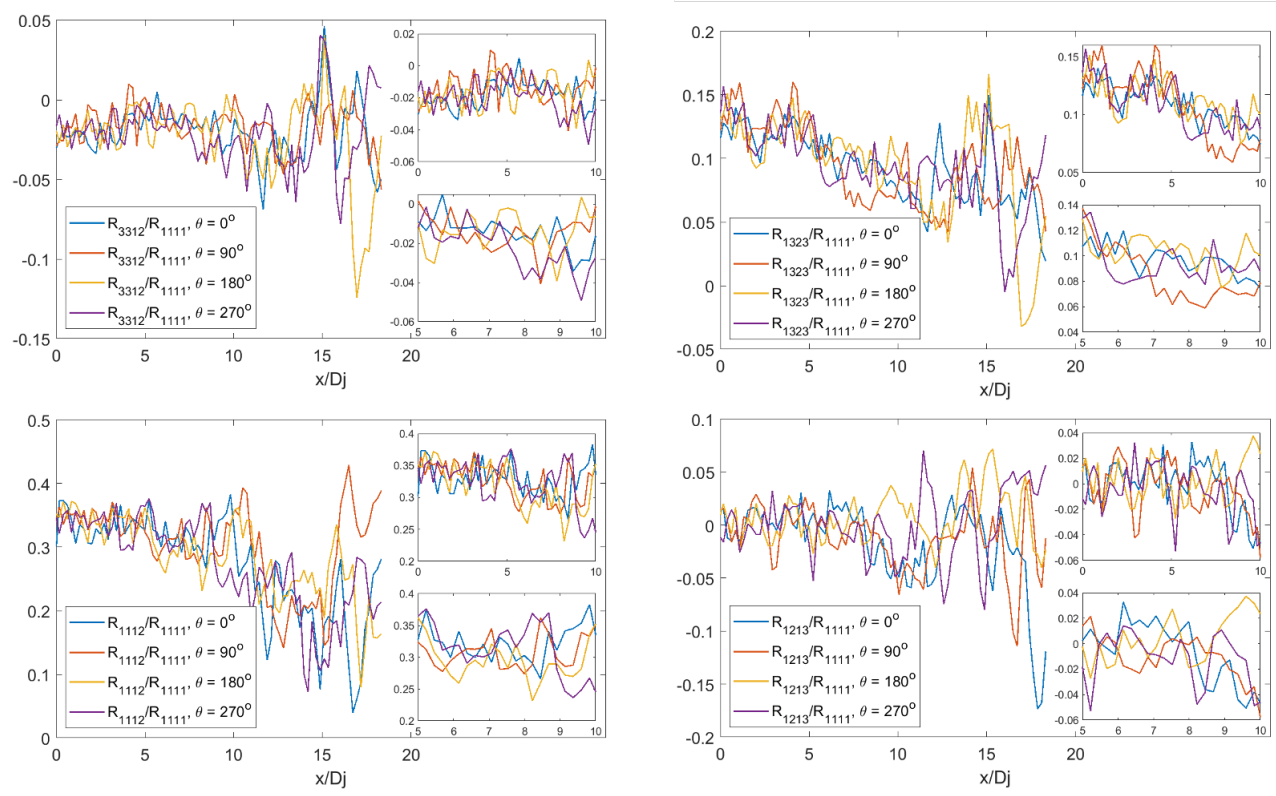

Fig. 14 Axial variation of extra components $\left(R_{3312}, R_{1323}, R_{1112}, R_{1213}\right.$ at 4 azimuthal planes: $\psi=$ $\left(0^{\circ}, 90^{\circ}, 180^{\circ}, 270^{\circ}\right)$ for $\mathrm{OP} 1.3$ at the bypass shear layer radial locations $r / D_{j}=0.38$ respectively.

[16] Ditlevsen, P. D., Turbulence and Shell Models, chapter and pages.

[17] Afsar, M. Z., Sescu, A., and Leib, S. J., "Modelling and prediction of the peak-radiated sound in subsonic axisymmetric air jets using acoustic analogy-based asymptotic analysis," Phil. Trans. R. Soc. A, Vol. 377, 2019.

[18] Batchelor, G. K., The theory of homogeneous turbulence, chapter and pages.

[19] Carrier, G. F., Krook, M., and Pearson, C. E., Functions of a Complex Variable, chapter and pages.

[20] B.Noble, Methods Based on the Wiener-Hopf Technique, chapter and pages.

[21] Leib, S. J., and Goldstein, M. E., “Hybrid Source Model for Predicting High-Speed Jet Noise,” AIAA J., Vol. 49, 2011 , p. 1324.

[22] Morse, P. M., and Feshbach, H., Methods of Theoretical Physics, Vol. I, chapter and pages.

[23] Bowman, F., Introduction to Bessel Functions, chapter and pages.

[24] Mohan, N. K. D., Dowling, A. P., Karabasov, S. A., Xia, H., Graham, O., Hynes, T. P., and Tucker, P. G., "Acoustic sources and far-field noise of chevron and round jets," AIAA J., Vol. 53, 2015, p. 2421.

[25] Karabasov, S. A., Bogey, C., and Hynes, T. P., "An investigation of the mechanisms of sound generation in initially laminar subsonic jets using the Goldstein acoustic analogy," J. Fluid Mech., Vol. 714, 2013, p. 24. 

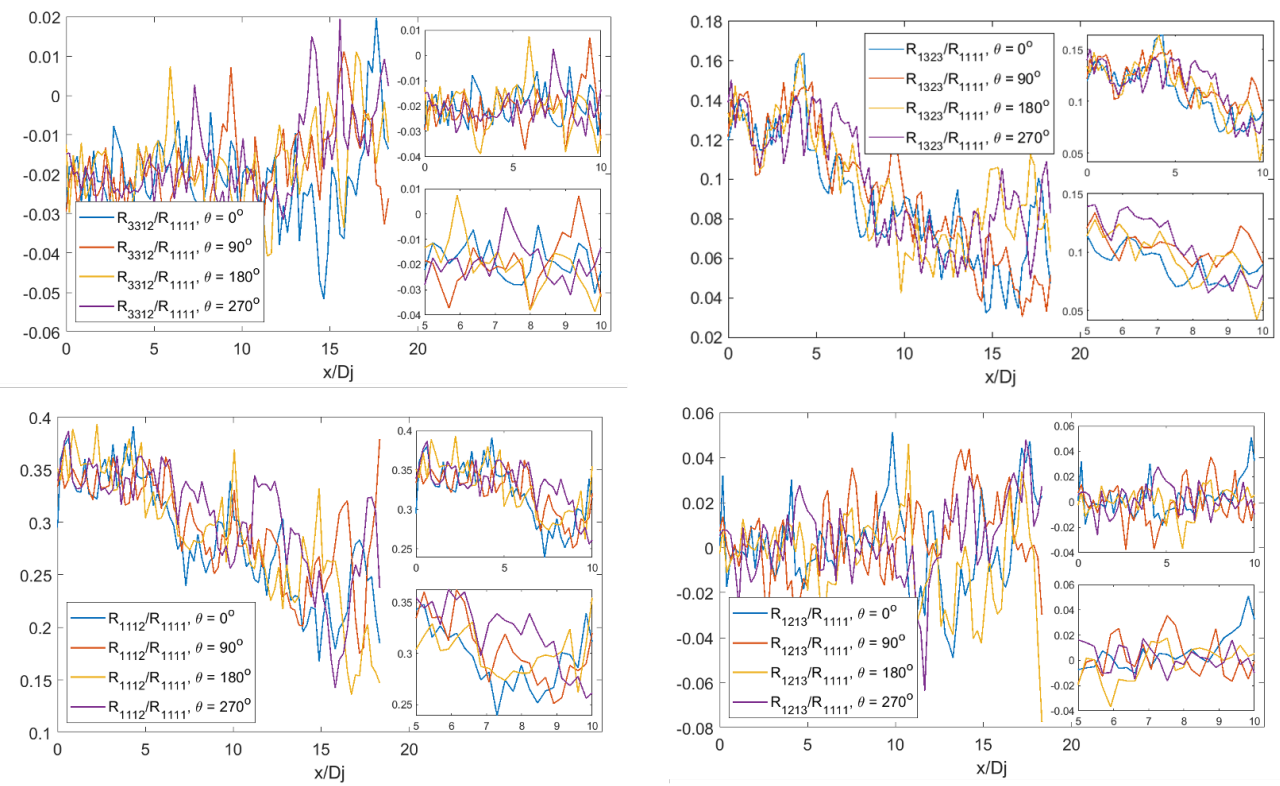

Fig. 15 Axial variation of extra components $\left(R_{3312}, R_{1323}, R_{1112}, R_{1213}\right.$ at 4 azimuthal planes: $\psi=$ $\left(0^{\circ}, 90^{\circ}, 180^{\circ}, 270^{\circ}\right)$ for $\mathrm{OP} 1.7$ at the bypass shear layer radial locations $r / D_{j}=0.38$ respectively.
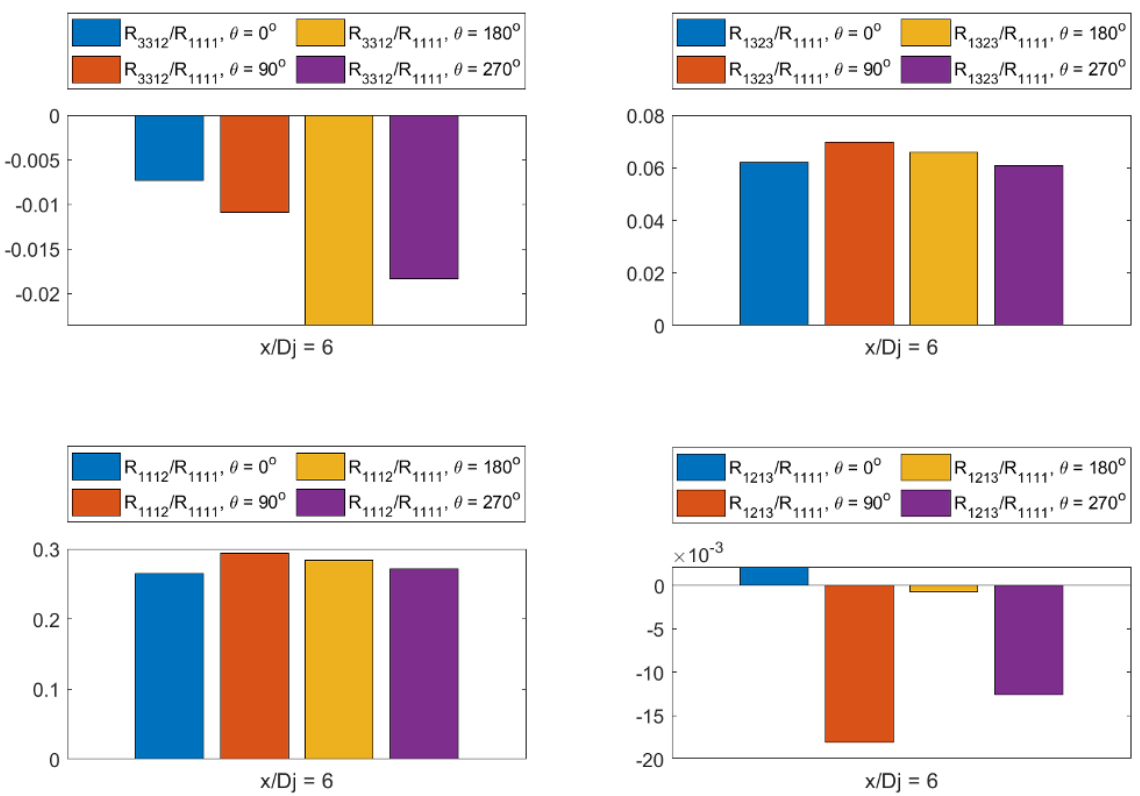

Fig. 16 Variation of extra components $\left(R_{3312}, R_{1323}, R_{1112}, R_{1213}\right)$ at 4 azimuthal planes: $\psi=$ $\left(0^{\circ}, 90^{\circ}, 180^{\circ}, 270^{\circ}\right)$ for $\mathrm{OP} 1.3$ at the core shear layer radial locations $r / D_{j}=0.18$. 

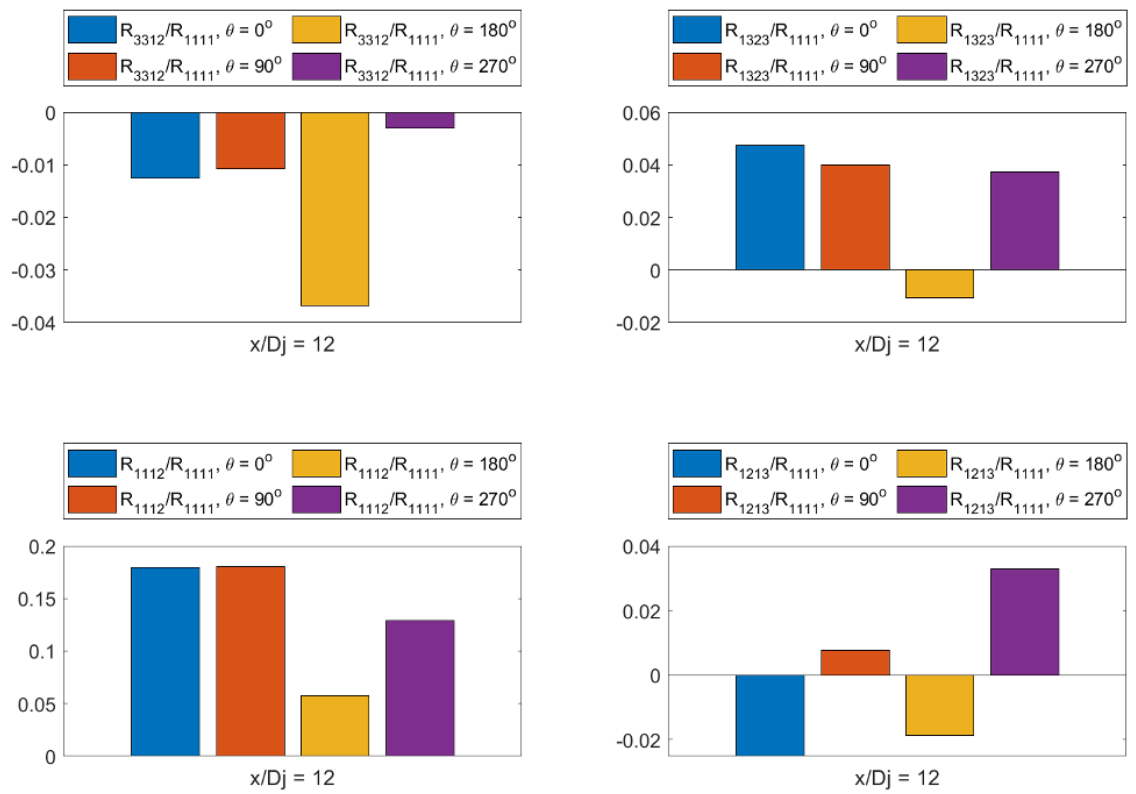

Fig. 17 Variation of extra components $\left(R_{3312}, R_{1323}, R_{1112}, R_{1213}\right)$ at 4 azimuthal planes: $\psi=$ $\left(0^{\circ}, 90^{\circ}, 180^{\circ}, 270^{\circ}\right)$ for $\mathrm{OP} 1.3$ at the core shear layer radial locations $r / D_{j}=0.18$.
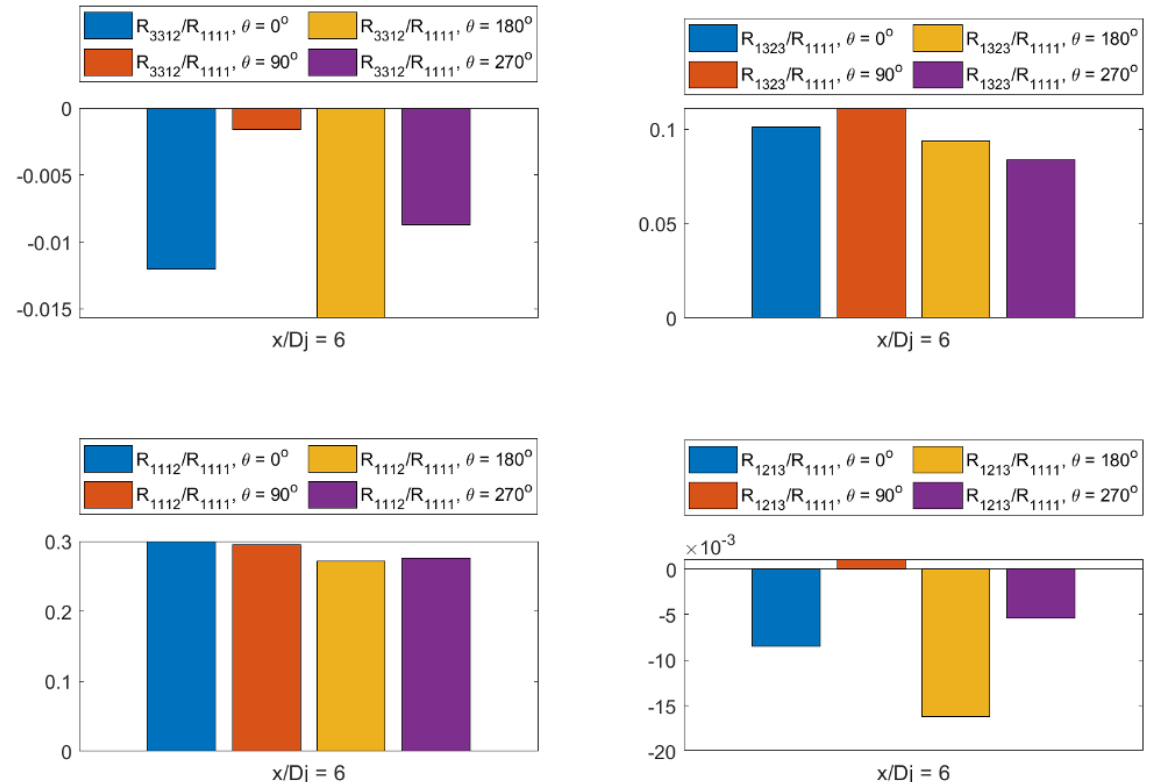

Fig. 18 Variation of extra components $\left(R_{3312}, R_{1323}, R_{1112}, R_{1213}\right)$ at 4 azimuthal planes: $\psi=$ $\left(0^{\circ}, 90^{\circ}, 180^{\circ}, 270^{\circ}\right)$ for $\mathrm{OP} 1.3$ at the bypass shear layer radial locations $r / D_{j}=0.38$. 

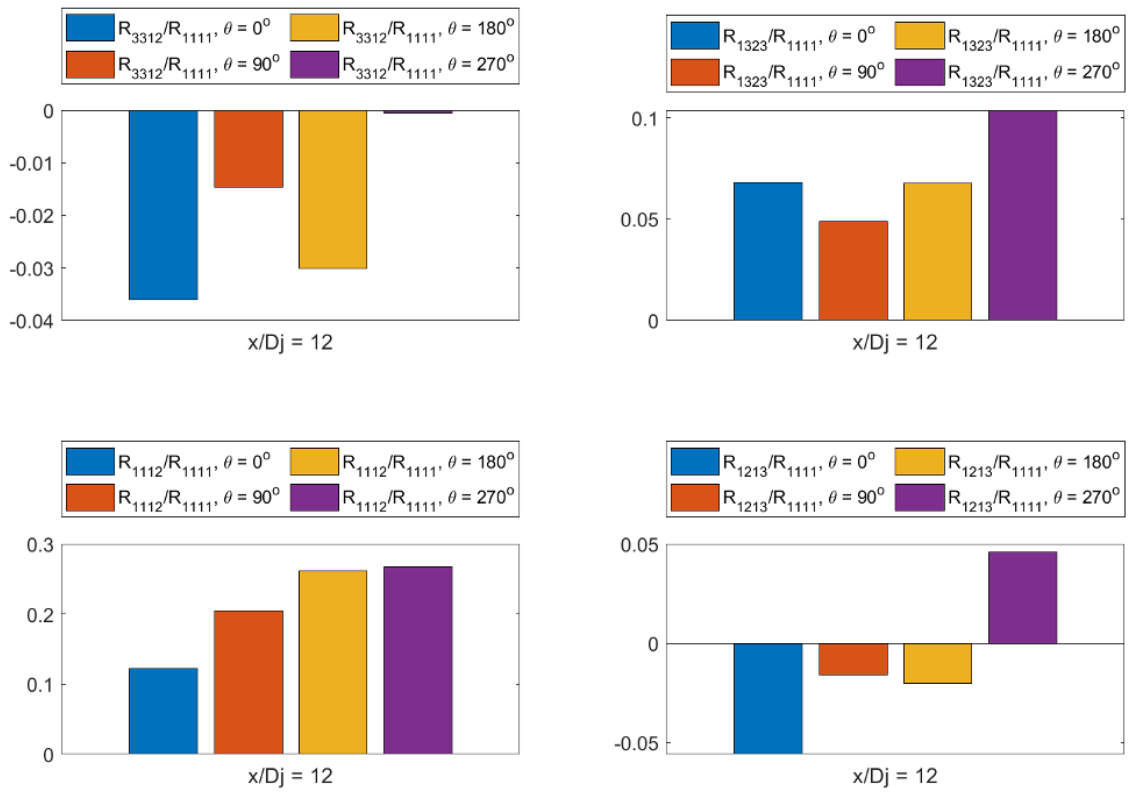

Fig. 19 Variation of extra components $\left(R_{3312}, R_{1323}, R_{1112}, R_{1213}\right)$ at 4 azimuthal planes: $\psi=$ $\left(0^{\circ}, 90^{\circ}, 180^{\circ}, 270^{\circ}\right)$ for $\mathrm{OP} 1.3$ at the bypass shear layer radial locations $r / D_{j}=0.38$.
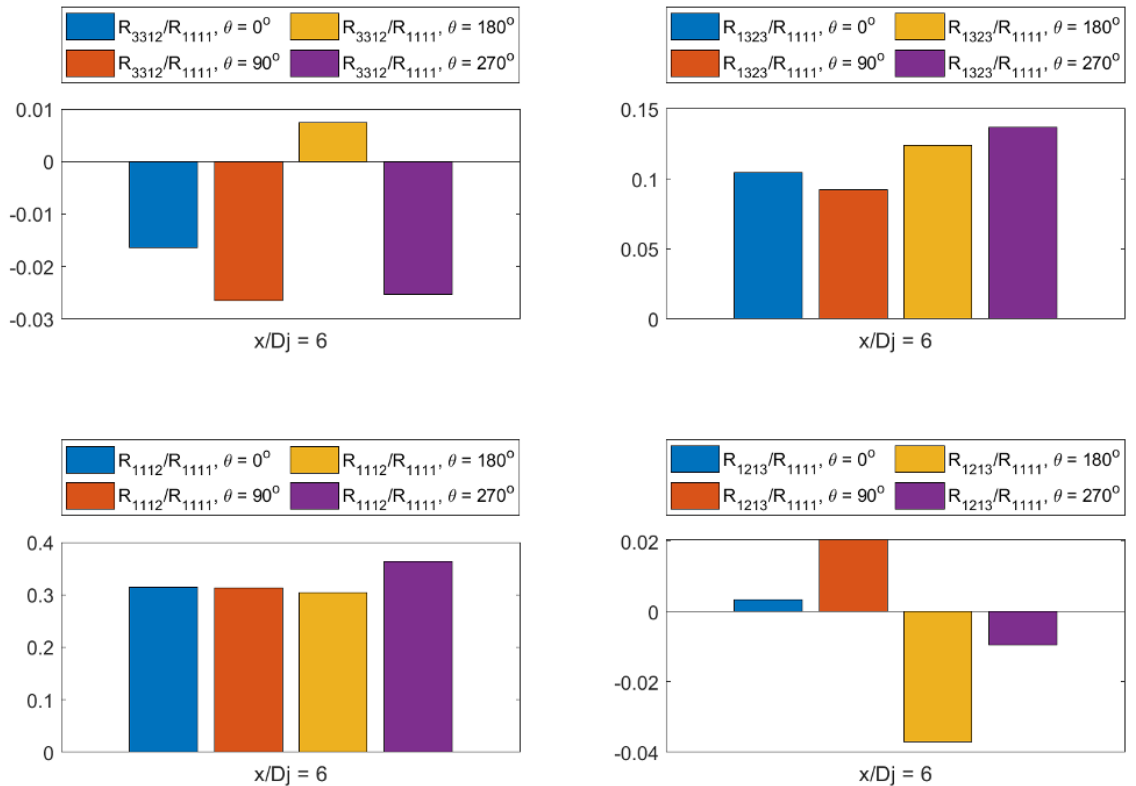

Fig. 20 Variation of extra components $\left(R_{3312}, R_{1323}, R_{1112}, R_{1213}\right)$ at 4 azimuthal planes: $\psi=$ $\left(0^{\circ}, 90^{\circ}, 180^{\circ}, 270^{\circ}\right)$ for $\mathrm{OP} 1.7$ at the bypass shear layer radial locations $r / D_{j}=0.38$. 

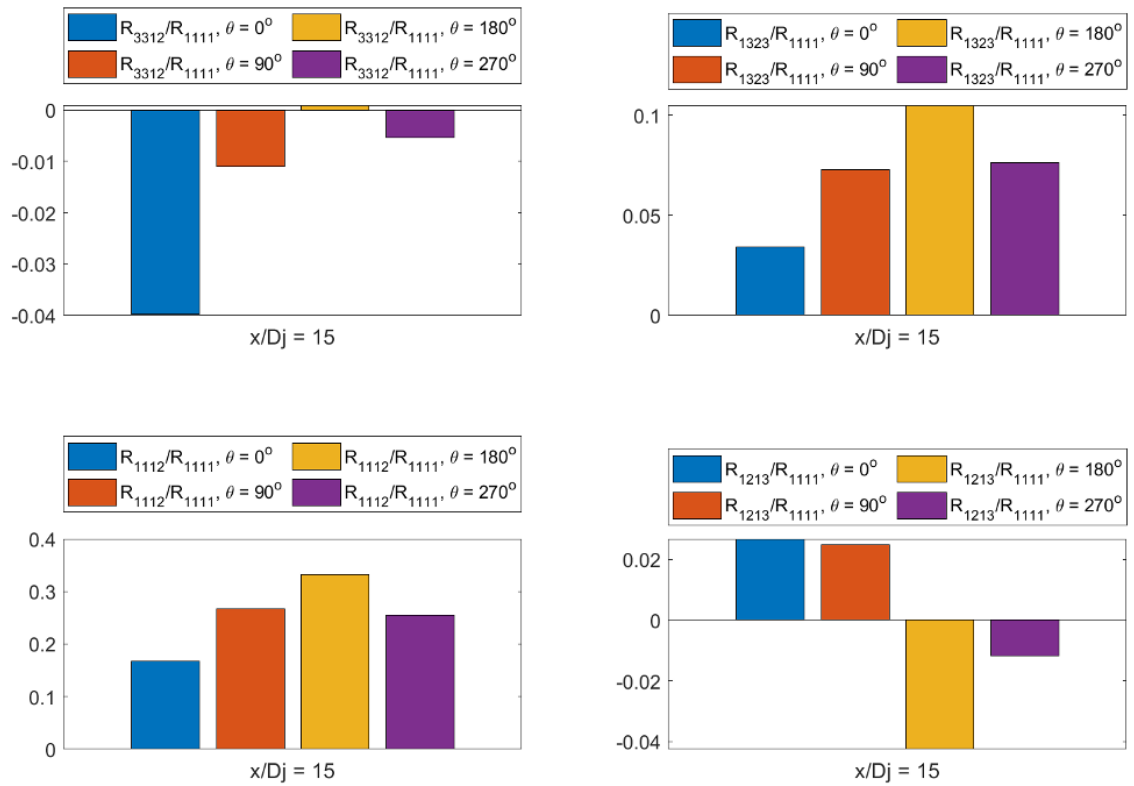

Fig. 21 Variation of extra components $\left(R_{3312}, R_{1323}, R_{1112}, R_{1213}\right)$ at 4 azimuthal planes: $\psi=$ $\left(0^{\circ}, 90^{\circ}, 180^{\circ}, 270^{\circ}\right)$ for $\mathbf{O P} 1.7$ at the bypass shear layer radial locations $r / D_{j}=0.38$.
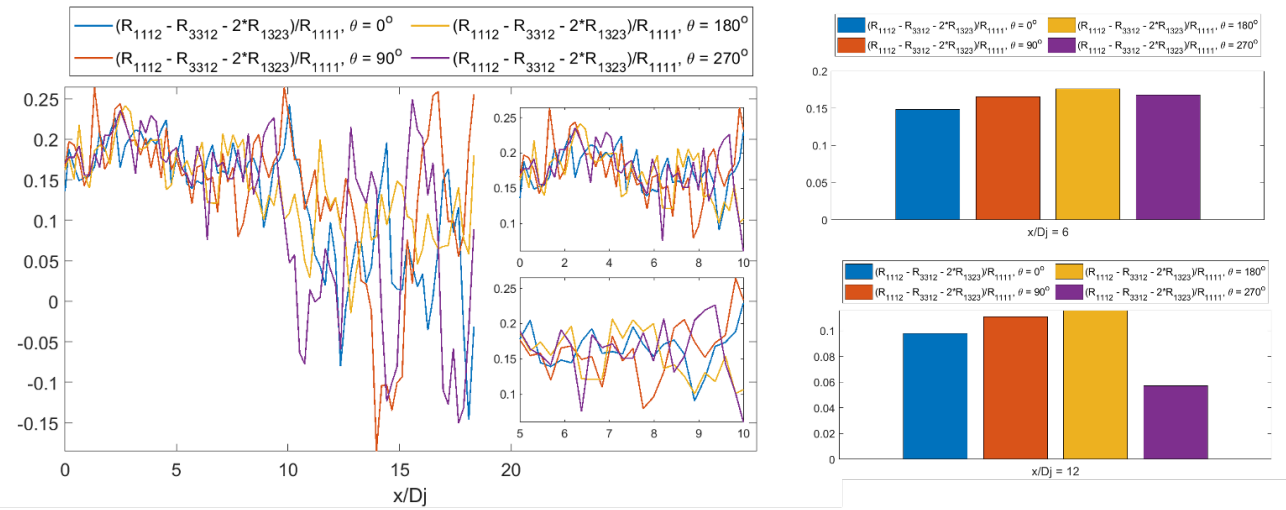

Fig. 22 Variation of term in $\left(R_{1112}-R_{3312}-2 R_{1323}\right)$ in two-term expansion formula $\left.29 \& 30\right)$ at 4 azimuthal planes: $\psi=\left(0^{\circ}, 90^{\circ}, 180^{\circ}, 270^{\circ}\right)$ for $\mathrm{OP} 1.3$ at the core shear layer radial locations $r / D_{j}=0.18$. 

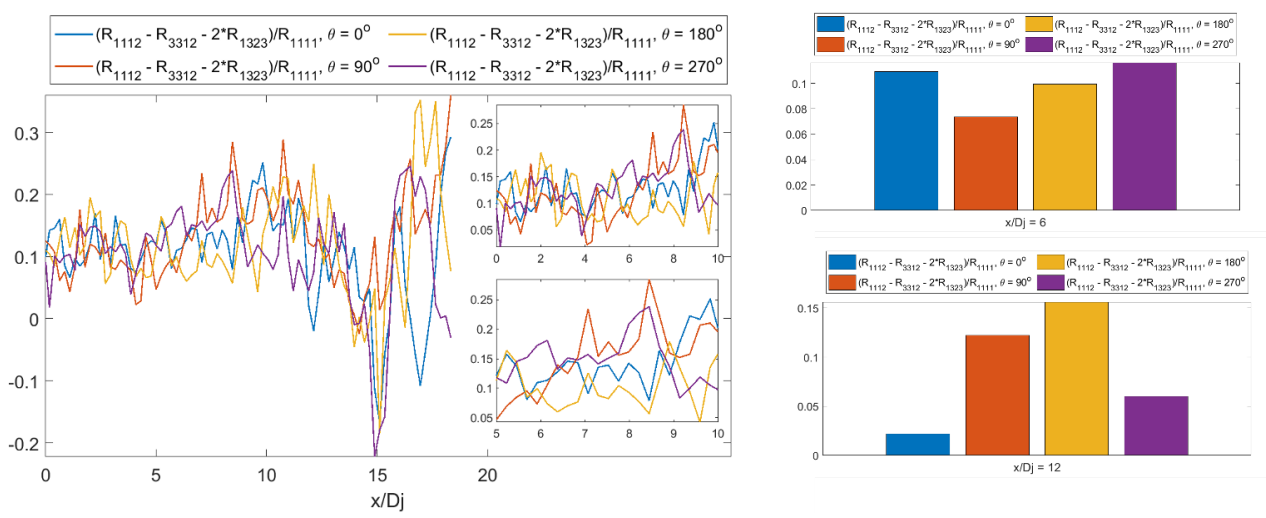

Fig. 23 Variation of term in $\left(R_{1112}-R_{3312}-2 R_{1323}\right)$ in two-term expansion formula $\left.29 \& 30\right)$ at 4 azimuthal planes: $\psi=\left(0^{\circ}, 90^{\circ}, 180^{\circ}, 270^{\circ}\right)$ for $\mathrm{OP} 1.3$ at the bypass shear layer radial locations $r / D_{j}=0.38$.
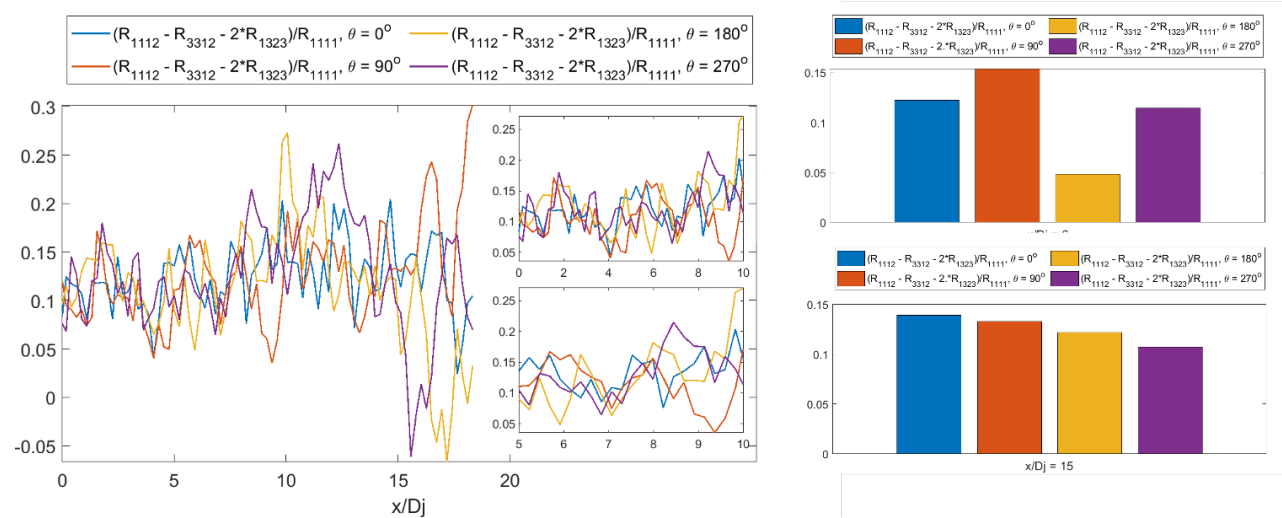

Fig. 24 Variation of term in $\left(R_{1112}-R_{3312}-2 R_{1323}\right)$ in two-term expansion formula 29 \& 30 at 4 azimuthal planes: $\psi=\left(0^{\circ}, 90^{\circ}, 180^{\circ}, 270^{\circ}\right)$ for $\mathrm{OP} 1.7$ at the bypass shear layer radial locations $r / D_{j}=0.38$.
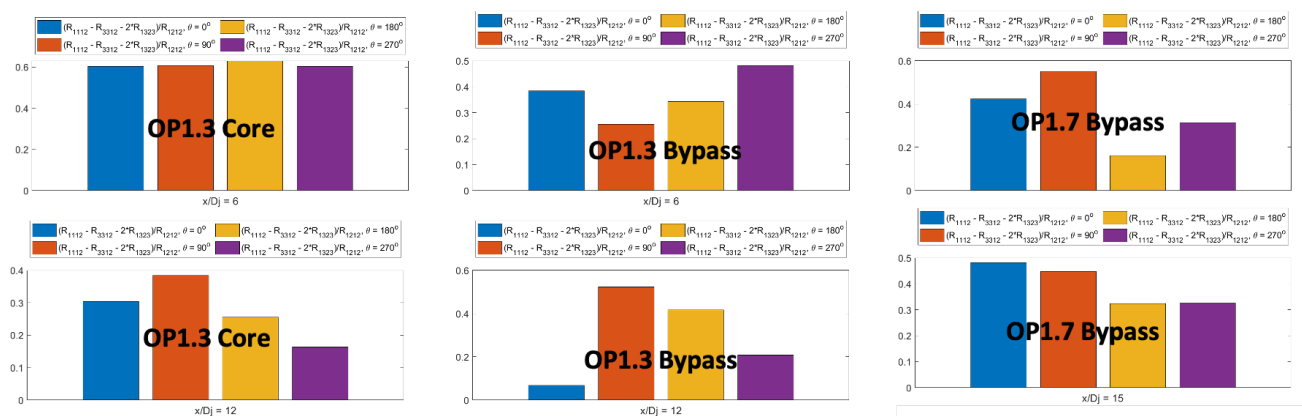

Fig. 25 Variation of term in $\left(R_{1112}-R_{3312}-2 R_{1323}\right)$ normalized by $R_{1212}$ for the two-term expansion formula 29 \& 30) at 4 azimuthal planes: $\psi=\left(0^{\circ}, 90^{\circ}, 180^{\circ}, 270^{\circ}\right)$ for $\mathrm{OP} 1.3 \&$ OP 1.7 at the core/bypass shear layer radial locations $\left(r / D_{j}=0.18,0.38\right)$ respectively. 